\title{
The systematic value of nuclear genome size for "all" species of Tulipa L. (Liliaceae)
}

\author{
Ben J. M. Zonneveld
}

Received: 2 December 2008 / Accepted: 8 June 2009/Published online: 11 July 2009

(C) The Author(s) 2009. This article is published with open access at Springerlink.com

\begin{abstract}
Nuclear genome size, as measured by flow cytometry with propidium iodide, was used to investigate the relationships within the genus Tulipa L. (Liliaceae). More than 400 accessions representing 123 taxa from mainly wild-collected plants were investigated. Most species of Tulipa have the same basic chromosome number, $2 n=2 x=24$. However, the somatic DNA $2 C$ value $(2 C)$ is shown to range from 32 to $69 \mathrm{pg}$ for the diploids. The largest genome contains roughly $3.4 \times 10^{10}$ more base pairs than the smallest and has chromosomes that are more than twice as large. These large differences in the amount of nuclear DNA predict that the hybrids, if any arise, are usually sterile. Depending on the size of the total genome, $1 \mathrm{pg}$ amounts to several thousand genes. Moreover, genome sizes are evaluated here in combination with available morphological, geographical, and molecular data. Therefore, the taxonomy proposed here is not a single-character taxonomy based on genome size alone. The genus Tulipa, as here determined, has 87 species, 29 more than accepted by van Raamsdonk et al. [Acta Hort (ISHS) 430:821-828, 1997], but including 25 species that were not available to them. Of these 87 species, 28 were not seen by Hall (The genus Tulipa, The Royal Horticultural Society, London, 1940) in a living state and placed by him in an addendum.
\end{abstract}

\footnotetext{
B. J. M. Zonneveld

Institute for Molecular Plant Sciences,

Clusius Laboratory, Leiden University,

P.O. Box 9505, 2300 RA Leiden, The Netherlands

e-mail: B.J.M.Zonneveld@biology.leidenuniv.nl

Present Address:

B. J. M. Zonneveld ( $\square)$

Nationaal Herbarium Nederland, P.O. Box 9514,

2300 RA Leiden, The Netherlands

e-mail: Zonneveld@NHN.leidenuniv.nl
}

Species of the subgenus Clusianae (Baker) Zonn. differ strongly in nuclear DNA content (DNA $2 C$ value), 32 versus 40-68 pg for all other tulips, and are placed here in a separate subgenus. Also Orithyia, the only group with a style and with only $38-39$ pg is placed in a separate subgenus. Therefore, all tulips are attributed to four subgenera, Clusianae (Baker) Zonn., Tulipa, Eriostemones Raamsd., and Orithyia (D. Don) Baker and divided further into 12 sections. Seven of the eight series of section Eichleres (A.D. Hall) Raamsd. are now placed in four sections: (1) section Lanatae (Raamsd.) Zonn., mainly confined to species from the Pamir-Alay and including series Lanatae Raamsd., (2) section Multiflorae (Raamsd.) Zonn. (including series Glabrae Raamsd.), (3) section Vinistriatae (Raamsd.) Zonn. (including series Undulatae Raamsd.), and (4) section Spiranthera Vved. ex Zonn. and Veldk. Triploids, tetraploids, and pentaploids were found in several species. DNA content confirmed the close relationships of the species within the different sections. The rather similar looking and therefore often confused T. armena Boiss. (51.8 pg), T. systola Stapf (56.3 pg), and T. julia K., Koch $(61.6 \mathrm{pg})$ could be clearly distinguished. The same is true for T. biebersteiniana Schult. f. (56.9 pg), T. sylvestris ssp. australis (Link) Pamp. (62.0 pg), and T. primulina Baker (64.6 pg). T. doerfleri Gand. and T. whittalli (Dykes) Hall could be placed as polyploid forms of T. orphanidea Boiss. ex Heldr. On the basis of DNA content, a systematic association between $T$. julia $\mathrm{K}$. Koch and the triploid T. aleppensis Boiss. and between T. systola Stapf and the triploid $T$. praecox Tenore was suggested. The new species T. lemmersii Zonn., Peterse, and de Groot is described, and four possible new species are indicated. Genome size as measured by using flow cytometry may conveniently be used to produce systematic data. It is applicable even in the case of dormant bulbs or sterile plants for monitoring the trade in bulbous species. 
Keywords Tulipa species - Genome size $\cdot C$-values . Taxonomy

\section{Introduction}

The genus Tulipa L. (Liliaceae) comprises 40 (Stork 1984) to 55 (van Raamsdonk et al. 1997) to more than 100 species (Hall 1940). In the World Checklist for Tulipa (Govaerts 2008), 418 are named and 112 were accepted there. Tulips occur naturally in southern Europe, North Africa, the Middle East, and central Asia, including China. The Tien Shan and Pamir-Alay mountain ranges in central Asia are considered the primary gene centers for Tulipa species (Botschantzeva 1962), with the Caucasus as a secondary center. They are popular spring-flowering garden plants; millions of bulbs are sold annually and over 5,000 cultivars are registered (van Scheepen 1996). Several species are in cultivation, but they cover less than $7 \%$ of the total tulip area in the Netherlands. The boundaries between taxa of various ranks are still a subject of dispute (van Raamsdonk et al. 1997). All species counted (with exception of the polyploid forms and T. maximowiczii Regel) have the same chromosome number, $2 n=2 x=24$ (De Mol 1928; Woods and Bamford 1937; Plavcova 1969). To elucidate the relationships between Tulipa species, the classical taxonomic traits based on morphological characters and geographical distribution are here supplemented with data on DNA $2 C$-values. These were not investigated earlier to any extent in the systematic study of Tulipa. In the list of DNA $C$-values (Bennett and Leitch 2004), data on 23 accessions of tulips were recorded, but these are hard to interpret as they come from different sources and methods, and they are intrinsically inconsistent.

Despite the existence of a large body of literature on Tulipa, taxonomy is generally considered to be difficult. Sentences to this effect are encountered in every taxonomic treatment but are especially applicable in this case. The main reason is that there is hardly any character that is not variable within a species. The main characteristics are as follows, but not a single one is without exceptions: leaf/ stem hairiness, a black blotch at the base of the tepals with or without a yellow edge, the presence and type of hairs on the inside of the bulb tunic, flower color, flowering time, and hairs on the base of the filaments. To circumvent this, van Raamsdonk (1992) and van Raamsdonk and De Vries (1995) used principal component and canonical variate analyses to investigate the variation of 35 morphological characters, and they substantially clarified systematic relationships within Tulipa. In this study, DNA $2 C$-value (nuclear DNA content) is introduced as an additional method for the study of Tulipa taxonomy. More than 400 different accessions representing nearly all accepted species were measured in an attempt to understand the relationships within Tulipa better.

Nuclear DNA content can conveniently be measured by flow cytometry using propidium iodide, a stoichiometric DNA stain that intercalates in the double helix. Where many species in a genus have the same chromosome numbers, differences in DNA $2 C$-value, when present, have proven to be very effective in delimiting infrageneric divisions in a number of taxa (Ohri 1998). Flow cytometry can therefore be considered as a fast and useful method for understanding systematic relationships. Genome size has been demonstrated to differ between taxa with identical chromosome numbers. Moreover, Greilhuber (1998, 2005) has clearly shown that intraspecific variation of genome size is much less than assumed.

The evolution of genome size ( $C x$ value; Greilhuber 1979) has received increased attention during recent years. Primitive angiosperms are now believed to have had small genomes; increases up to a factor of 1,000 have occurred independently in various modern taxa (Leitch et al. 1998). Flow cytometry has successfully been used to measure the $2 C$ value for the genera Hosta Tratt., Helleborus L., Clivia Lindl., Nerine Herb., Agapanthus L'Her., Galanthus L., Narcissus L., Gasteria Duval., and others by Zonneveld et al. (2001-2008). In this paper it is shown, using several accessions for most species, that many species can be discriminated based on their genome size and intraspecific variation is low in most Tulipa species. Details of the revised nomenclature for the infrageneric taxa are submitted by Veldkamp and Zonneveld.

\section{Materials and methods}

\section{Plant material}

Plant material was obtained from the collections of the Bulb Research Center of the Koninklijke Algemeene Vereeniging voor Bloembollencultuur (KAVB), Lisse via E. Breed; W. Lemmers, Lisse; J. van Tuyl, Wageningen University; J. de Groot, de Zilk; J.P. Tyssen, M. van den Brink, and J. Zonneveld (Hortus Bulborum, Limmen), all in the Netherlands; L. Lieser, France; J. Ruksans, Latvia; G. Knoche, Germany; and H. and M. Aanesen, Norway. Where possible, material of known wild origin was used, and care was taken to ensure correct identification of all material. Vouchers of most species are in the Herbarium of Wageningen University (WAG) and the Royal Botanic Garden, Kew, UK (K). 
Flow cytometric measurement of nuclear DNA content

For the isolation of nuclei, about $0.5 \mathrm{~cm}^{2}$ of adult leaf tissue was chopped together with a piece of Agave americana L. 'Aureomarginata' or Clivia miniata (Lindl.) Regel as an internal standard (see below). The chopping was done with a new razor blade in a Petri dish in $0.25 \mathrm{ml}$ nucleiisolation buffer to which $0.25 \mathrm{mg}$ RNase $/ \mathrm{ml}$ was added (Zonneveld and Van Iren 2001). After adding $1.75 \mathrm{ml}$ propidium iodide solution (50 mg PI/l in isolation buffer), the suspension with nuclei was filtered through a $30-\mu \mathrm{m}$ nylon filter. The fluorescence of the nuclei was measured $30 \mathrm{~min}$ and $1 \mathrm{~h}$ after addition of propidium iodide, using a Partec CA-II flow cytometer. The optical path contained a HBO mercury lamp, filters KG1 and BG12, dichroic mirror TK500, filter OG570, and a Leitz $50 \times 1$ water immersion objective. Data were analyzed by means of DPAC software (Partec). The $2 C$ DNA content of the sample was calculated as the sample peak mean, divided by the Agave/Clivia peak mean, and multiplied by the amount of DNA of the Agave/Clivia standard. At least three different samples, with at least 5,000 nuclei each, were measured twice for each clone. Most histograms revealed a coefficient of variation of less than $5 \%$. The standard deviation was calculated for the DNA content of each species, using all relevant measurements.

\section{Internal standard and absolute DNA content values}

When measuring nuclear DNA content by means of flow cytometry, it is necessary to chop tissue from the plant of interest together with an internal standard: this standard must be as close as possible to the plants of interest. In this way, variation in signal intensities due to staining kinetics, to light absorption and quenching by sample components, as well as to instrument and other variables, is reduced to a minimum. Agave americana was chosen as internal standard for tulips of subgenus Clusianae (Baker) Zonn. and section Kolpakowskianae Raamsd. ex Zonn. and Veldk. (including their polyploids) with about $32-45 \mathrm{pg}$ of DNA for the diploids; Clivia miniata was used for all other tulips. Clivia miniata and A. americana are available year-round, do not mind several weeks without water and, being large plants, a single specimen can serve a lifetime, thereby further reducing variation in readings. They also have a low background in propidium iodide measurements and show a single $G_{0}$ peak, almost lacking $G_{2}$ arrest. Fresh male human leucocytes $\left(2 C=7.0 \mathrm{pg} ; 1 \mathrm{pg}=10^{-12}\right.$ gram $= \pm 10^{9}$ bp) were chosen as primary standard (Tiersch et al. 1989). This yields $2 C=15.9 \mathrm{pg}$ for nuclei of Agave americana L. and $39.0 \mathrm{pg}$ for Clivia miniata.

\section{Results and discussion}

\section{Genome size}

Tulips, as with most species that arouse a strong horticultural interest, are not easy to investigate as it is often difficult to discriminate between wild tulips and escaped ones. Moreover, very few characters are constant in tulips. Even with a strong character such as hairs on the filaments that discriminate between subgenus Eriostemones Raamsd. and all other tulips, species are found among bonafide Eriostemones where the hairs are absent, e.g., in T. sprengeri Baker and T. sogdiana Bunge. Another important character is the number and shape of the hairs inside the bulb tunic. Here also differences are found within a population (Botschantzeva 1962), and moreover seem also to depend strongly on conditions of cultivation. Flower color is often of minor importance, as especially in the Tien Shan (Pratov et al. 2006) and adjacent desert areas, several species, including $T$. schrenkii Regel, T. alberti Regel, T. greigii Regel, T. kaufmanniana Regel, and T. ostrowskiana Regel, range from white, yellow, and orange to red colors. Even in species with mainly red flowers, being most prominent in the Pamir-Alay, an occasional yellow (edged) flower can be found. Furthermore a yellow margin to a black blotch on the base of a tepal, even the blotch itself, can be present or absent within a species (Van Raamsdonk and De Vries 1995). Genome size as investigated here (see Table 1), complements the work based mainly on morphological characters of Hall (1940) and of morphological characters, crossability studies and geographical distribution of van Raamsdonk et al. (1991-1997). Although van Raamsdonk et al. used about 35 characters to discriminate among the species, they remark that some species come out identical in their scheme yet can be distinguished by characters not used in their investigations. Our start was to see how few species we could distinguish; Van Raamsdonk et al. (1997) with 55 species seemed a very good basis, but in the end, we could not be avoid restoring several more taxa to species status. Moreover, 25 additional species could be investigated that were not available to Van Raamsdonk and De Vries (1995).

\section{Subdivision of the genus Tulipa}

Traditionally genus Tulipa is divided in two sections: Tulipa (syn.: Leiostemones Boiss.) and Eriostemones. Van Raamsdonk et al. (1997) raise these to subgenus status and divide the subgenus Tulipa into five sections: Clusianae Baker, Kopalkowskiana (A.D. Hall) Raamsd., Eichleres (A.D. Hall) Raamsd., Tulipanum Reboul, and Tulipa. Section Eichleres was further divided into eight series. Section Eriostemones is simpler. Since Hall (1940), most 
Table 1 All species of Tulipa with their amount of DNA 2C, average, standard deviation, place of origin, and the names under which the clones were received

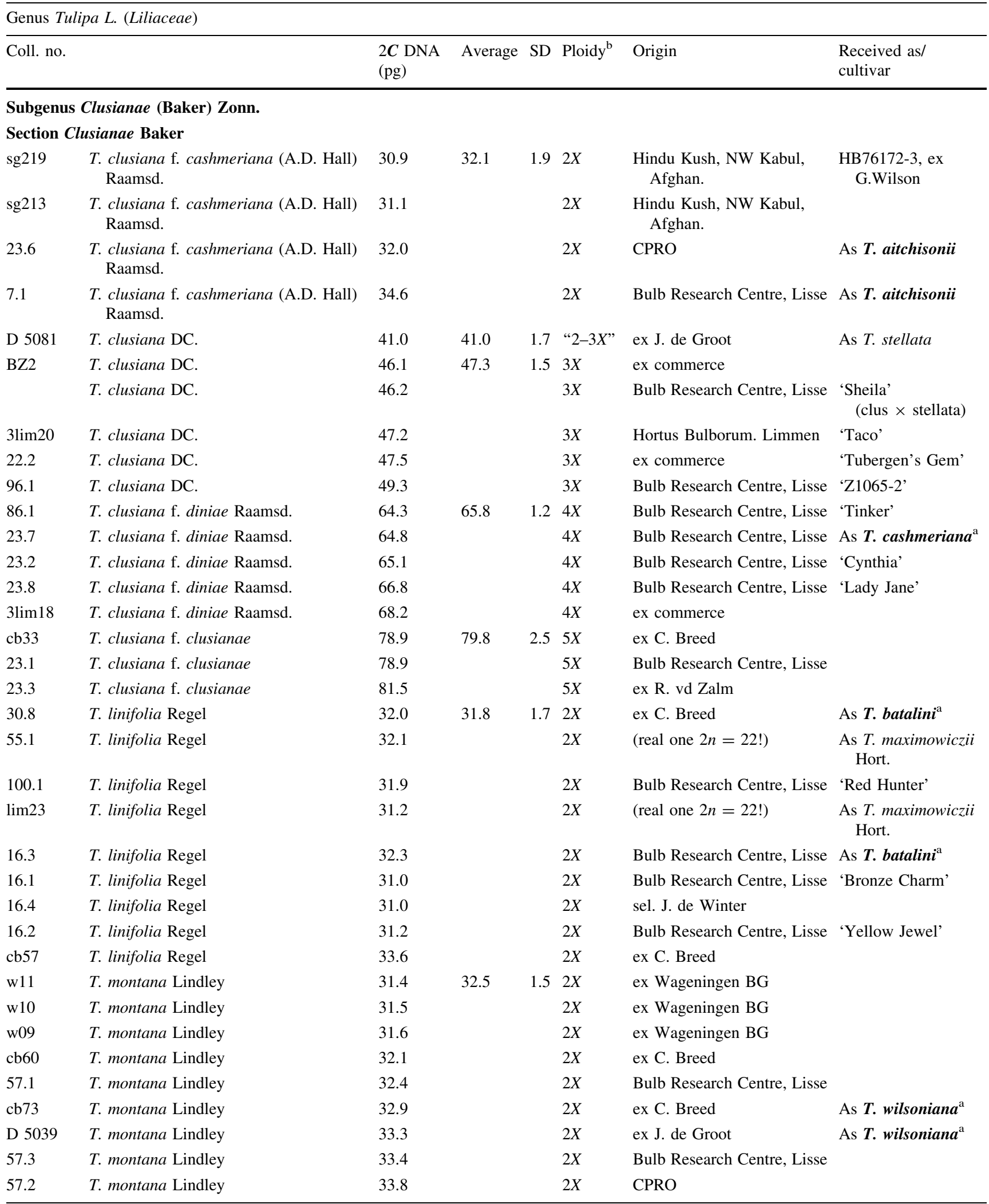


Table 1 continued

Genus Tulipa L. (Liliaceae)

\begin{tabular}{llll}
\hline Coll. no. & $\begin{array}{l}2 \boldsymbol{C} \text { DNA Average SD Ploidy } \\
(\mathrm{pg})\end{array}$ & Origin & $\begin{array}{l}\text { Received as/ } \\
\text { cultivar }\end{array}$ \\
\hline
\end{tabular}

Subgenus Orithyia (D.Don) Baker

Section Orithyia (D.Don) Vved.

\begin{tabular}{|c|c|c|c|c|c|c|c|}
\hline $\operatorname{sg} 12 / 4$ & T. heterophylla (Regel) Baker & 37.5 & 37.5 & 0.3 & $2 X$ & $\begin{array}{l}\text { Tien Shan, N. Almaty, } \\
\text { Kazak. }\end{array}$ & On acid soil \\
\hline $\operatorname{sg} 8 / 5$ & T. uniflora (L.) Besser ex Baker & 38.3 & 38.3 & 0.4 & $2 X$ & W. Tarbagatai, Kazakhstan & \\
\hline testc & T. heteropetala Ledeb. & 38.4 & 39.1 & 0.6 & $2 X$ & Altai, Kazakhstan & \\
\hline $\operatorname{sg} 314 \mathrm{c}$ & T. heteropetala Ledeb. & 39.2 & & & $2 X$ & $\begin{array}{l}\text { Altai, Marble Pass, } \\
\text { Kazakhstan }\end{array}$ & \\
\hline sg314a & T. heteropetala Ledeb. & 39.4 & & & $2 X$ & $\begin{array}{l}\text { Altai, Chin. border, } \\
\text { Kazakhstan }\end{array}$ & \\
\hline $\operatorname{sg} 314 b$ & T. heteropetala Ledeb. & 39.4 & & & $2 X$ & $\begin{array}{l}\text { Altai, E Irtich river, } \\
\text { Kazakhstan }\end{array}$ & \\
\hline
\end{tabular}

Subgenus Tulipa

Section Kolpakowskianae Raamsd. ex Zonn \& Veldk.

112-1 T. lemmersii Zonn., A. Peterse, J. de Groot sp.nov.

112-2 T. lemmersii Zonn., A. Peterse, J. de Groot sp. 36.4 nov.

BZ08

T. nitida $\mathrm{Hoog}$

T. nitida Hoog

BZ07 T. zenaidae Vved.

$97.1 \quad$ T. zenaidae Vved.

T. zenaidae Vved.

$42.2 T$ T. iliensis Regel

testc.

T. iliensis Regel

81 T. iliensis Regel

D5303

T. iliensis Regel

$42.1 \quad T$. iliensis Regel

D5403b T. iliensis Regel

D 5080a T. iliensis Regel

D 5080b T. iliensis Regel

D5403d T. iliensis Regel

sg T. iliensis Regel

D5420 T. brachystemon Regel

BZ07 T. brachystemon Regel

sg T. brachystemon Regel

D5419 T. brachystemon Regel

sg T. brachystemon Regel

sg T. brachystemon Regel

BZ07 T. korolkowii Regel

T. korolkowii Regel

BZ08 T. korolkowii Regel f. rosea Zonn.

BZ07 T. korolkowii Regel f. rosea Zonn.

D 5136

T. borszczowii Baker

T. kolpakowskiana Baker

sg211 T. kolpakowskiana Baker
38.3

38.9

39.3

39.7

40.5

39.4

40.3

40.3

40.8

\section{4}

36

$\begin{array}{lrl} & & 2 X \\ 38.6 & 0.8 & 2 X \\ & & 2 X \\ 39.8 & 1.0 & 2 X \\ & & 2 X \\ & & 2 X \\ 41.0 & 0.9 & 2 X \\ & & 2 X\end{array}$

$2 X$

$2 X$

$2 X$

$1.02 X$

$2 X$

$2 X$

$0.92 X$

$2 X$

$2 X$

$2 X$

$2 X$

$2 X$

$2 X$

$2 X$

$2 X$

42.0

62.1

39.3

40.9

41.6

41.7

41.6

40.7

40.6

41.0

42.1

42.2

41.6

39.2

40.0

$62.1 \quad 1.23 X$

$\begin{array}{lll}41.0 & 0.9 & 2 X\end{array}$

$\begin{array}{lll}41.5 & 0.9 \quad 2 X\end{array}$

$41.6 \quad 1.4 \quad 2 X$

$\begin{array}{lll}41.8 & 1.5 & 2 X\end{array}$
Kazakhstan

Mashad Pass, Chimkent, Kaz.

Mashad Pass, Chimkent, Kaz.

Dehqanabad, Uzbekistan

Dehqanabad, Uzbekistan

Merke valley, Kazakhstan

Bulb Research Centre, Lisse

Merke Valley, Kazakhstan

Bulb Research Centre, Lisse

Bulb Research Centre, Lisse As $T$.

Bulb Research Centre, Lisse

HB77268

Bulb Research Centre, Lisse

ex J. de Groot

Marni 01, China

Ketmen Range, Kazakhstan

HB74247

commerce

Dzjungaric foothills,

$$
\text { Kazakhstan }
$$

Kazakhstan

Kazakhstan

Kazakhstan

Ili River

Dzjungaric Ala-Tau

Mogoltau, Uzbekistan

Iron Gate, Uzbekistan

Turkestansky range

As $T$. rosea $a^{a}$

Turkestansky range

As $T$. rosea $a^{a}$

Kyzyl-kum Lake, Kazakhstan

Charyn Canyon, Kazakhstan 
Table 1 continued

\begin{tabular}{|c|c|c|c|c|c|c|c|}
\hline \multicolumn{8}{|c|}{ Genus Tulipa L. (Liliaceae) } \\
\hline Coll. no. & & $\begin{array}{l}2 C \text { DNA } \\
(\mathrm{pg})\end{array}$ & Average & SD & Ploidy ${ }^{b}$ & Origin & $\begin{array}{l}\text { Received as/ } \\
\text { cultivar }\end{array}$ \\
\hline \multirow[t]{2}{*}{ D5400 } & T. kolpakowskiana Baker & 40.9 & & & $3 X$ & Kazakhstan & \\
\hline & T. kolpakowskiana Baker & 41.0 & & & $2 X$ & Merke Valley, Kazakhstan & \\
\hline \multirow[t]{3}{*}{ BZ07 } & T. kolpakowskiana Baker & 41.3 & & & $2 X$ & Near Ili River, Kazakhstan & \\
\hline & T. kolpakowskiana Baker & 41.5 & & & $2 X$ & Merke Valley, Kazakhstan & \\
\hline & T. kolpakowskiana Baker & 42.0 & & & $2 X$ & Kabchagal, Kazakhstan & As $T$. alberti small \\
\hline 59.2 & T. kolpakowskiana Baker & 42.1 & & & $2 X$ & CPRO 82337 & As T. neustruevae \\
\hline D 5021a & T. kolpakowskiana Baker & 43.2 & & & $2 X$ & fr coll seed JJA02, Kazak. & \\
\hline $\operatorname{cb} 68$ & T. kolpakowskiana Baker & 44.3 & & & $2 X$ & ex C. Breed & \\
\hline D5415 & T. lehmanniana Merckl. & 42.0 & 42.4 & 1.0 & $2 X$ & & As T. behmiana ${ }^{\text {a }}$ \\
\hline BZ07 & T. lehmanniana Merckl. & 42.1 & & & $2 X$ & Kabchagal Kazakhstan no 1 & As T. behmiana ${ }^{\text {a }}$ \\
\hline \multirow[t]{2}{*}{ BZ07 } & T. lehmanniana Merckl. & 42.7 & & & $2 X$ & Kabchagal Kazakhstan no 2 & As T. behmiana ${ }^{\text {a }}$ \\
\hline & T. lehmanniana Merckl. & 42.7 & & & $2 X$ & Badhyz, Turkmenistan & \\
\hline 52.1 & T. lehmanniana Merckl. & 67.6 & 67.6 & & $3 X$ & CPRO 77161, ex M. Hoog & \\
\hline D 5066a & T. hissarica Popov \& Vved. & 42.8 & 42.8 & 1.3 & $2 X$ & Hodji-obi-garm, Tajikistan & \\
\hline D 5066 & T. hissarica Popov \& Vved. & 42.8 & & & $2 X$ & ex P.Christian 1999. & \\
\hline testc. & T. anisophylla Vved. & 42.0 & 43.4 & 1.2 & $2 X$ & Bulb Research Centre, Lisse & \\
\hline 5.2 & T. anisophylla Vved. & 43.5 & & & $2 X$ & Bulb Research Centre, Lisse & \\
\hline 5.1 & T. anisophylla Vved. & 44.4 & & & $2 X$ & CPRO76333-2,BG Dunshanbe & \\
\hline \multirow[t]{2}{*}{47.1} & T. korshinskyi Vved. & 43.5 & 44.0 & 0.7 & $2 X$ & Bulb Research Centre, Lisse & \\
\hline & T. korshinskyi Vved. & 44.5 & & & $2 X$ & Darwas Mnt, Z. Tadjikistan & \\
\hline D 5041 & T. ferganica Vved. & 44.3 & 44.8 & 1.8 & $2 X$ & ex J. de Groot & \\
\hline cb44 & T. ferganica Vved. & 44.7 & & & $2 X$ & ex C. Breed & \\
\hline D5405a & T. ferganica Vved. & 45.6 & & & $2 X$ & HB80291-1, d yellow & \\
\hline $\operatorname{sg} 208$ & T. altaica Pall. ex Spreng. & 43.2 & 44.9 & 1.6 & $2 X$ & Altai 2005 , broad lf & \\
\hline $\operatorname{sg} 210$ & T. altaica Pall. ex Spreng. & 43.6 & & & $2 X$ & Marble pass, Altai 2004 & \\
\hline $\operatorname{sg} 9 / 5$ & T. altaica aff. Pall. ex Spreng. & 44.5 & & & $2 X$ & Dzjungaric Ala-Tau & \\
\hline 2008 & T. altaica Pall. ex Spreng. & 44.7 & & & $2 X$ & Saur Mountains, Kazakhstan & \\
\hline D5401b & T. altaica Pall. ex Spreng. & 44.7 & & & $2 X$ & ex J. de Groot & \\
\hline \multirow[t]{2}{*}{ testc. } & T. altaica Pall. ex Spreng. & 45.5 & & & $2 X$ & Altai, Kazakhstan & \\
\hline & T. altaica Pall. ex Spreng. & 45.5 & & & $2 X$ & Altai, Kazakhstan & \\
\hline 2008 & T. altaica Pall. ex Spreng. & 45.7 & & & $2 X$ & W. Tarbagatai, Kazakhstan & \\
\hline testc. & T. altaica Pall. ex Spreng. & 46.5 & & & $2 X$ & Marble Pass, Altai 2005 & \\
\hline D5403a & T. ostrowskiana Regel & 76.7 & 77.7 & 1.9 & $4 X$ & Chu \& Ily Mnts & \\
\hline D5407 & T. ostrowskiana Regel & 77.1 & & & $4 X$ & ex J. de Groot,HB78120-0 & \\
\hline D5400 & T. ostrowskiana Regel & 77.6 & & & $4 X$ & HB93194-1 & \\
\hline 84.3 & T. ostrowskiana Regel & 77.7 & & & $4 X$ & Bulb Research Centre, Lisse & \\
\hline 84.1 & T. ostrowskiana Regel & 79.4 & & & $4 X$ & Bulb Research Centre, Lisse & \\
\hline $\operatorname{sgl}$ & T. tetraphylla Baker & 39.0 & 40.1 & 1.5 & $2 X$ & W. China & \\
\hline sgl & T. tetraphylla Baker & 41.1 & & & $2 X$ & Ketman Range, ZO. Kazakhstan & \\
\hline $\operatorname{cb} 31$ & T. tetraphylla Baker & 77.7 & 79.9 & 1.4 & $4 X$ & ex C. Breed & \\
\hline \multirow[t]{2}{*}{84.2} & T. tetraphylla Baker & 78.5 & & & $4 X$ & CPRO 93151 & \\
\hline & T. tetraphylla Baker & 78.8 & & & $4 X$ & Kyrgyzstan & \\
\hline 84.7 & T. tetraphylla Baker & 79.6 & & & $4 X$ & CPRO 93149 & \\
\hline 17642 & T. tetraphylla Baker & 80.4 & & & $4 X$ & Ene of Kordai 1,800 m, Kazak. & \\
\hline 4.1 & T. tetraphylla Baker & 80.6 & & & $4 X$ & Bulb Research Centre, Lisse & As $T$. altaica \\
\hline
\end{tabular}


Table 1 continued

\begin{tabular}{|c|c|c|c|c|c|c|c|}
\hline \multicolumn{8}{|c|}{ Genus Tulipa L. (Liliaceae) } \\
\hline \multicolumn{2}{|l|}{ Coll. no. } & \multirow{2}{*}{$\begin{array}{l}\begin{array}{l}2 C \text { DNA } \\
(\mathrm{pg})\end{array} \\
81.0\end{array}$} & \multirow[t]{2}{*}{ Average } & \multirow[t]{2}{*}{ SD } & \multirow{2}{*}{$\begin{array}{l}\text { Ploidy }^{\mathrm{b}} \\
4 X\end{array}$} & \multirow{2}{*}{$\begin{array}{l}\text { Origin } \\
\text { HB } 87136\end{array}$} & \multirow{2}{*}{$\begin{array}{l}\begin{array}{l}\text { Received as/ } \\
\text { cultivar }\end{array} \\
\text { As } T . \text { sp. }\end{array}$} \\
\hline D 5099 & T. tetraphylla Baker & & & & & & \\
\hline 4.2 & T. tetraphylla Baker & 82.5 & & & $4 X$ & Bulb Research Centre, Lisse & As $T$. altaica \\
\hline \multicolumn{8}{|c|}{ Section Multiflorae (Raamsd.) Zonn. } \\
\hline 81.1 & T. subpraestans Vved. & 46.8 & 45.7 & 1.2 & $2 X$ & Bulb Research Centre, Lisse & \\
\hline $\operatorname{cb} 05$ & T. subpraestans Vved. & 44.6 & & & $2 X$ & ex C. Breed & \\
\hline 41.2 & T. heweri Raamsd. & 46.9 & 47.7 & 1.2 & $2 X$ & Bulb Research Centre, Lisse & Short form \\
\hline $2 \lim 26$ & T. heweri Raamsd. & 47.6 & & & $2 X$ & Hortus Bulborum, Limmen & \\
\hline 41.1 & T. heweri Raamsd. & 47.9 & & & $2 X$ & Bulb Research Centre, Lisse & Long form \\
\hline cb09 & T. heweri Raamsd. & 48.3 & & & $2 X$ & ex C. Breed & \\
\hline $2 \lim 33$ & T. praestans Hoog & 48.8 & 50.1 & 0.6 & $2 X$ & Hortus Bulborum, Limmen & 'Princess Red Sun' \\
\hline $2 \lim 32$ & T. praestans Hoog & 49.1 & & & $2 X$ & Hortus Bulborum, Limmen & ‘Princess Shogon’ \\
\hline 69.2 & T. praestans Hoog & 49.7 & & & $2 X$ & Bulb Research Centre, Lisse & ‘Tubergen's var’ \\
\hline CB68 & T. praestans Hoog & 50.7 & & & $2 X$ & Bulb Research Centre, Lisse & 'Fuselier' \\
\hline 69.1 & T. praestans Hoog & 51.0 & & & $2 X$ & Bulb Research Centre, Lisse & 'Zwaneburg'(wild coll.) \\
\hline CB67 & T. praestans Hoog & 51.0 & & & $2 X$ & Bulb Research Centre, Lisse & ‘Unicum’ \\
\hline \multicolumn{8}{|c|}{ Section Lanatae (Raamsd.) Zonn. comb. et stat. nov. } \\
\hline CB70 & T. hoogiana B.Fedtsch. & 47.9 & 48.8 & 1.2 & $2 X$ & ex C. Breed & \\
\hline CB207 & T. hoogiana B.Fedtsch. & 48.0 & & & $2 X$ & ex C. Breed & \\
\hline $\operatorname{cb} 15$ & T. hoogiana B.Fedtsch. & 48.4 & & & $2 X$ & ex C. Breed & \\
\hline $\operatorname{sg} 310$ & T. hoogiana B.Fedtsch. & 49.3 & & & $2 X$ & ex J. de Groot & \\
\hline 39.1 & T. hoogiana B.Fedtsch. & 50.6 & & & $2 X$ & Bulb Research Centre, Lisse & \\
\hline sg & T. hoogiana B.Fedtsch. & 73.6 & 73.6 & & $3 X$ & ex J. de Groot & \\
\hline 32.1 & $T . \mathrm{sp}$. & 49.7 & 50.8 & 0.9 & $2 X$ & Bulb Research Centre, Lisse & As T. eichleri horT. \\
\hline $\operatorname{cb} 06$ & $T . \mathrm{sp}$. & 50.5 & & & $2 X$ & ex C. Breed & As T. eichleri horT. \\
\hline 32.3 & T. sp. & 51.1 & & & $2 X$ & Bulb Research Centre, Lisse & As T. eichleri horT. \\
\hline 32.2 & T. sp. & 51.3 & & & $2 X$ & Bulb Research Centre, Lisse & As T. eichleri horT. \\
\hline D5205 & T. sp. & 51.4 & & & $2 X$ & ex J. de Groot & 'Clare Benedict' \\
\hline $\mathrm{cb} 12$ & T. fosteriana Hoog & 51.2 & 51.9 & 1.0 & $2 X$ & ex C. Breed & 'Rockery Beauty' \\
\hline $\operatorname{cb} 10$ & T. fosteriana Hoog & 51.5 & & & $2 X$ & ex C. Breed & 'Madame Lefeber' \\
\hline cb11 & T. fosteriana Hoog & 51.9 & & & $2 X$ & ex C. Breed & 'Cantata' \\
\hline 99.1 & T. fosteriana Hoog & 51.9 & & & $2 X$ & Bulb Research Centre, Lisse & 'Mrs. Dagnia' \\
\hline D $5004 \mathrm{a}$ & T. fosteriana Hoog & 52.0 & & & $2 X$ & Seravschan, Uzbekistan & \\
\hline $\operatorname{cb} 13$ & T. fosteriana Hoog & 53.2 & & & $2 X$ & ex C. Breed & \\
\hline $\operatorname{sg} 224$ & T. affinis Botschantz. & 52.4 & 52.5 & 1.3 & $2 X$ & ex J. de Groot & \\
\hline 3.1 & T. affinis Botschantz. & 52.6 & & & $2 X$ & Bulb Research Centre, Lisse & \\
\hline 50.1 & T. lanata Regel & 52.1 & 52.5 & 0.4 & $2 X$ & CPRO 65369, ex v Tubergen & \\
\hline $\operatorname{sg} 220$ & T. lanata Regel & 51.9 & & & $2 X$ & ex J. de Groot & \\
\hline Lim19 & T. lanata Regel & 53.4 & & & $2 X$ & Hortus Bulborum, Limmen & \\
\hline 50.2 & T. lanata Regel & 78.9 & 78.9 & 0.1 & $3 X$ & No. 1256 & \\
\hline 89.4 & T. tubergeniana Hoog & 52.4 & 53.5 & 1.1 & $2 X$ & Bulb Research Centre, Lisse & 'Splendens' \\
\hline $\operatorname{cb} 03$ & T. tubergeniana Hoog & 52.5 & & & $2 X$ & ex C. Breed & As $T$. ingens ${ }^{\mathrm{a}}$ \\
\hline 89.1 & T. tubergeniana Hoog & 53.9 & & & $2 X$ & CPRO 65391, ex v Tubergen & \\
\hline 43.1 & T. tubergeniana Hoog & 54.0 & & & $2 X$ & Bulb Research Centre, Lisse & As $T$. ingens $^{\mathrm{a}}$ \\
\hline $\operatorname{sg} 106$ & T. tubergeniana Hoog & 54.0 & & & $2 X$ & Baisun, Uzbekistan & \\
\hline 89.3 & T. tubergeniana Hoog & 54.1 & & & $2 X$ & Bulb Research Centre, Lisse & \\
\hline D $5055 a$ & T. carinata Vved. & 53.9 & 54.2 & 1.2 & $2 X$ & Sangardak Valley, Uzbekistan & \\
\hline
\end{tabular}


Table 1 continued

\begin{tabular}{|c|c|c|c|c|c|c|c|}
\hline \multicolumn{8}{|c|}{ Genus Tulipa L. (Liliaceae) } \\
\hline \multicolumn{2}{|l|}{ Coll. no. } & \multirow{2}{*}{$\begin{array}{l}2 C \text { DNA } \\
(\mathrm{pg})\end{array}$} & \multirow[t]{2}{*}{ Average } & \multirow[t]{2}{*}{$\mathrm{SD}$} & \multirow{2}{*}{$\frac{\text { Ploidy }^{\mathrm{b}}}{2 X}$} & \multirow{2}{*}{$\begin{array}{l}\text { Origin } \\
\text { Sangardak Valley, Uzbekistan }\end{array}$} & \multirow[t]{2}{*}{$\begin{array}{l}\text { Received as/ } \\
\text { cultivar }\end{array}$} \\
\hline $\mathrm{BZ}$ & T. carinata Vved. & & & & & & \\
\hline \multicolumn{8}{|c|}{ Section Vinistriatae (Raamsd.) Zonn. } \\
\hline $\operatorname{cb} 75$ & T. micheliana Hoog & 47.4 & 48.0 & 0.8 & $2 X$ & ex C. Breed & \\
\hline $\operatorname{sg} 302$ & T. micheliana Hoog & 48.2 & & & $2 X$ & ex J. de Groot & \\
\hline D 5114 & T. micheliana Hoog & 48.7 & & & $2 X$ & Kopet Dagh, Cambridge B03 & \\
\hline $\operatorname{sg} 226$ & T. micheliana Hoog & 49.2 & & & $2 X$ & ex J. de Groot & \\
\hline sg & T. micheliana Hoog & 47.4 & & & $2 X$ & Kopet Dagh, Iran & \\
\hline sg & T. micheliana Hoog & 47.3 & & & $2 X$ & Kopet Dagh, Iran & \\
\hline 93.1 & T. vvedenskyi Botschantz. & 50.2 & 51.4 & 1.7 & $2 X$ & Bulb Research Centre, Lisse & \\
\hline 93.3 & T. vvedenskyi Botschantz. & 50.4 & & & $2 X$ & Bulb Research Centre, Lisse & \\
\hline 8.1 & T. vvedenskyi Botschantz. & 50.8 & & & $2 X$ & Bulb Research Centre, Lisse & \\
\hline 93.4 & T. vvedenskyi Botschantz. & 51.0 & & & $2 X$ & CPRO 81139 & \\
\hline 93.2 & T. vvedenskyi Botschantz. & 51.3 & & & $2 X$ & CPRO large form & \\
\hline 93.5 & T. vvedenskyi Botschantz. & 51.4 & & & $2 X$ & Bulb Research Centre, Lisse & \\
\hline $093.7 ?$ & T. vvedenskyi Botschantz. & 51.5 & & & $2 X$ & Bulb Research Centre, Lisse & \\
\hline $\mathrm{cb} 02$ & T. vvedenskyi Botschantz. & 51.7 & & & $2 X$ & ex C. Breed & \\
\hline $\operatorname{cb} 20$ & T. vvedenskyi Botschantz. & 51.8 & & & $2 X$ & ex C. Breed & \\
\hline 93.8 & T. vvedenskyi Botschantz. & 51.9 & & & $2 X$ & Bulb Research Centre, Lisse & \\
\hline CB63 & T. vvedenskyi Botschantz. & 52.4 & & & $2 X$ & ex Potterton \& Martin & 'Hanka' \\
\hline \multirow[t]{2}{*}{ D5113 } & T. vvedenskyi Botschantz. & 52.7 & & & $2 X$ & Karakorum Mtns, Tadjikistan & \\
\hline & T. alberti Regel & 51.4 & 52.4 & 1.6 & $2 X$ & Kabchagal, Kazakhstan & \\
\hline D5323a & T. alberti Regel & 52.3 & & & $2 X$ & Kara-Tau, Kazakhstan & \\
\hline \multirow[t]{2}{*}{ D5323a } & T. alberti Regel & 52.3 & & & $2 X$ & Kara-Tau, Kazakhstan & \\
\hline & T. alberti Regel & 52.5 & & & $2 X$ & Kabchagal, Kazakhstan & \\
\hline BZ07 & T. alberti Regel & 53.3 & & & $2 X$ & Kabchagal, Kazakhstan & \\
\hline \multirow[t]{2}{*}{56.1} & T. mogoltavica Popov \& Vved. & 51.4 & 51.6 & 1.5 & $2 X$ & Mogoltau, Tadjikistan & \\
\hline & T. mogoltavica Popov \& Vved. & 51.7 & & & $2 X$ & Mogoltau, Tadjikistan & \\
\hline $\operatorname{cb} 01$ & T. greigii Regel & 52.2 & 53.4 & 1.0 & $2 X$ & ex C. Breed & \\
\hline \multirow[t]{2}{*}{$\operatorname{cb} 19$} & T. greigii Regel & 53.4 & & & $2 X$ & Dzambul desert & \\
\hline & T. greigii Regel & 53.5 & & & $2 X$ & Berkara Valley, Kazakhstan & \\
\hline BZ7 & T. greigii Regel & 54.7 & & & $2 X$ & Red Hill, Dzabagly, Kazakhstan & \\
\hline D 5115 & T. butkovii Botschantz. & 53.9 & 53.9 & 0.9 & $2 X$ & $\begin{array}{l}\text { Akbulak Valley, Chimgan, } \\
\text { Uzbek. }\end{array}$ & \\
\hline \multicolumn{8}{|c|}{ Section Spiranthera Vved. ex Zonn. \& Veldk. } \\
\hline $\operatorname{sg} 215$ & T. berkariensis Ruksans nom. ill. & 50.7 & 50.8 & 0.2 & $2 X$ & Kshi Kaindy, Kazakhstan & As T. kaufmanniana aff. \\
\hline $\operatorname{sg} 214$ & T. berkariensis Ruksans nom. ill. & 51.1 & & & $2 X$ & Ulken Kaindy, Kazakhstan & As T. kaufmanniana aff. \\
\hline \multirow[t]{3}{*}{ sgl } & T. berkariensis Ruksans nom. ill. & 50.7 & & & & Aksu-Djabagly Res. Kazakhstan & As T. kaufmanniana aff. \\
\hline & T. berkariensis Ruksans nom. ill. & 75.9 & 75.9 & 1.7 & $3 X$ & Kazakhstan & As T. kaufmanniana aff. \\
\hline & T. kaufmanniana Regel & 52.2 & 53.2 & 1.4 & $2 X$ & Tubergen selection & 'Gaiety' \\
\hline CB220 & T. kaufmanniana Regel & 52.7 & & & $2 X$ & type in general culture & \\
\hline $\operatorname{cb} 64$ & T. kaufmanniana Regel & 53.1 & & & $2 X$ & Urun Gachsai, Uzbekistan & \\
\hline CB218 & T. kaufmanniana Regel & 53.2 & & & $2 X$ & type ex P. Nyssen & \\
\hline $\operatorname{sg} 5$ & T. kaufmanniana Regel & 53.3 & & & $2 X$ & ex J. de Groot & \\
\hline D65318e & T. kaufmanniana Regel & 53.6 & & & $2 X$ & Urun Gachsai, Uzbekistan & \\
\hline $\operatorname{cb} 69$ & T. kaufmanniana Regel & 54.5 & & & $2 X$ & Ugan, Uzbekistan via J.Ruksans & 'Ice Sticks' \\
\hline 87.4 & T. tschimganica Botschantz. & 52.4 & 53.7 & 0.9 & $2 X$ & Bulb Research Centre, Lisse & Type 4 \\
\hline
\end{tabular}


Table 1 continued

\begin{tabular}{|c|c|c|c|c|c|c|c|}
\hline \multicolumn{8}{|c|}{ Genus Tulipa L. (Liliaceae) } \\
\hline Coll. no. & & $\begin{array}{l}2 \boldsymbol{C} \text { DNA } \\
(\mathrm{pg})\end{array}$ & Average & SD & Ploidy $^{\mathrm{b}}$ & Origin & $\begin{array}{l}\text { Received as/ } \\
\text { cultivar }\end{array}$ \\
\hline 87.2 & T. tschimganica Botschantz. & 54.0 & & & $2 X$ & Bulb Research Centre, Lisse & Type 2 \\
\hline 87.1 & T. tschimganica Botschantz. & 54.3 & & & $2 X$ & Bulb Research Centre, Lisse & Type 1 \\
\hline 87.3 & T. tschimganica Botschantz. & 54.3 & & & $2 X$ & Bulb Research Centre, Lisse & Type 3 \\
\hline D 5060 & T. dubia Vved. & 54.0 & 54.7 & 0.9 & $2 X$ & Chimgan, Uzbekistan & \\
\hline 31.2 & T. dubia Vved. & 54.0 & & & $2 X$ & Bulb Research Centre, Lisse & Type 2 \\
\hline $\operatorname{cb} 70$ & T. dubia Vved. & 54.5 & & & $2 X$ & Beldersai, Uzbekistan & \\
\hline 31.1 & T. dubia Vved. & 55.1 & & & $2 X$ & Bulb Research Centre, Lisse & Type 1 \\
\hline D 5059 & T. dubia Vved. & 55.9 & & & $2 X$ & Ruksans 1999, Beldersai & \\
\hline \multicolumn{8}{|c|}{ Section Tulipanum Reboul } \\
\hline $2 \lim 40$ & T. undulatifolia Boiss. & 51.2 & 51.2 & 0.5 & $2 X$ & Hortus Bulborum, Limmen & \\
\hline Aanesen & T. undulatifolia Boiss. & 76.8 & 76.8 & 0.8 & $3 X$ & Didyma, Peloponesus, Greece & As $T$. boeotica ${ }^{\text {a }}$ \\
\hline Knoche & T. cypria Stapf ex Turill & 78.8 & 78.8 & 0.8 & $3 X$ & Akamas peninsula, Cyprus & \\
\hline 62.1 & T. armena Boiss. & 50.8 & 51.8 & 1.7 & $2 X$ & Bulb Research Centre, Lisse & As species 2 fr Turkey \\
\hline $\operatorname{sg} 303$ & T. armena Boiss. & 51.1 & & & $2 X$ & ex J. de Groot & As species 1 fr Turkey \\
\hline 63.1 & T. armena Boiss. & 51.2 & & & $2 X$ & Bulb Research Centre, Lisse & As species 3 fr Turkey \\
\hline 60.1 & T. armena Boiss. & 51.2 & & & $2 X$ & Bulb Research Centre, Lisse & As T. oculus-solis, short \\
\hline 1.2 & T. armena Boiss. & 51.2 & & & $2 X$ & Bulb Research Centre, Lisse & CPRO 74223 \\
\hline 1.3 & T. armena Boiss. & 51.4 & & & $2 X$ & Bulb Research Centre, Lisse & \\
\hline tc 60.1 & T. armena Boiss. & 52.2 & & & $2 X$ & Bulb Research Centre, Lisse & As T. agenensis \\
\hline $\operatorname{sg} 221$ & T. armena Boiss. & 53.3 & & & $2 X$ & ex J. de Groot from Tsechie & 'Excelsa' \\
\hline 1.1 & T. armena Boiss. & 53.9 & & & $2 X$ & Bulb Research Centre, Lisse & CPRO88036 \\
\hline 1.4 & T. armena Boiss. & 75.5 & 75.5 & 0.8 & $3 X$ & Bulb Research Centre, Lisse & As $T$. armena ssp. lycica \\
\hline $\operatorname{sg} 110$ & T. kuschkensis Fedtsch. & 53.3 & 53.3 & 0.4 & $2 X$ & Turkmenistan & \\
\hline $5208 \mathrm{c}$ & T. stapfii Turill & 52.3 & 52.7 & 0.6 & $2 X$ & Kordestan, Iran & \\
\hline sg & T. stapfii Turill & 53.1 & & & & Khoshyeylag Pass, Iran & \\
\hline D 5079 & T. systola Stapf & 55.1 & 56.1 & 1.1 & $2 X$ & V.S. 2000 & As $T \cdot$ stapfii $^{\mathrm{a}}$ \\
\hline w02 & T. systola Stapf & 55.4 & & & $2 X$ & Wageningen $\mathrm{BG}$ & \\
\hline w04 & T. systola Stapf & 55.6 & & & $2 X$ & Wageningen $\mathrm{BG}$ & \\
\hline $\operatorname{sg} 56$ & T. systola Stapf & 55.8 & & & $2 X$ & ex J. de Groot & As T. ulophylla \\
\hline w03 & T. systola Stapf & 55.9 & & & $2 X$ & Wageningen BG & \\
\hline 88036 & T. systola Stapf & 56.2 & & & $2 X$ & Bulb Research Centre, Lisse & As $T . j u l i a$ \\
\hline w07 & T. systola Stapf & 56.3 & & & $2 X$ & Wageningen BG & \\
\hline D5105 & T. systola Stapf & 56.5 & & & $2 X$ & Erzurum, Turkey, ex BG Got. & As $T$. sintenisii \\
\hline w06 & T. systola Stapf & 56.7 & & & $2 X$ & Wageningen BG & \\
\hline 5079 & T. systola Stapf & 56.7 & & & $2 X$ & ex J. de Groot & As T.stapfii $i^{\mathrm{a}}$ \\
\hline w05 & T. systola Stapf & 57.2 & & & $2 X$ & Wageningen BG & \\
\hline $\operatorname{sgl}$ & T. schmidtii Fomin & 57.9 & 57.9 & 0.9 & $2 \mathrm{x}$ & W. of Calilabad S. Azerbaijan & \\
\hline D5202a & T. julia K. Koch & 60.4 & 61.6 & 2.0 & $2 X$ & Lebanon & As T. aleppensis \\
\hline $\operatorname{cb} 58$ & T. julia K. Koch & 61.0 & & & $2 X$ & ex Cambridge BG & \\
\hline D $5117 \mathrm{a}$ & T. julia K. Koch & 61.0 & & & $2 X$ & ex Seed ES01 & As $T$. arm. ssp lycica \\
\hline 101.1 & T. julia K. Koch & 61.3 & & & $2 X$ & Bulb Research Centre, Lisse & As T. stapfii \\
\hline cb61 & T. julia K. Koch & 61.5 & & & $2 X$ & ex C. Breed & As $T$. armena \\
\hline 44.2 & T. julia K. Koch & 63.2 & & & $2 X$ & Bulb Research Centre, Lisse & Long form \\
\hline 44.1 & T. julia K. Koch & 64.1 & & & $2 X$ & BG Yerevan, Armenia & CPRO 72119-16 \\
\hline sg & T. julia K. Koch & 62.4 & & & $2 X$ & Mt. Arai, N.Yerevan, Armenia & \\
\hline D5119 & T. praecox Tenore & 57.9 & 57.9 & 0.7 & $2 X$ & Salda Lake,W Turkey & Seed ES01 as $T$. ndulata \\
\hline
\end{tabular}


Table 1 continued

\begin{tabular}{|c|c|c|c|c|c|c|c|}
\hline \multicolumn{8}{|c|}{ Genus Tulipa L. (Liliaceae) } \\
\hline Coll. no. & & $\begin{array}{l}2 C \text { DNA } \\
(\mathrm{pg})\end{array}$ & Average & $\mathrm{SD}$ & Ploidy $^{b}$ & Origin & $\begin{array}{l}\text { Received as/ } \\
\text { cultivar }\end{array}$ \\
\hline w01 & T. praecox Tenore & 87.3 & 88.3 & 0.6 & $3 X$ & ex Wageningen BG & \\
\hline $\operatorname{sg} 7$ & T. praecox Tenore & 87.3 & & & $3 X$ & ex J. de Groot & \\
\hline tc 68,1 & T. praecox Tenore & 88.3 & & & $3 X$ & Bulb Research Centre, Lisse & \\
\hline tc 60,2 & T. praecox Tenore & 88.3 & & & $3 X$ & Bulb Research Centre, Lisse & As T. oculus-solis ${ }^{\mathrm{a}}$ \\
\hline $2 \lim 39$ & T. praecox Tenore & 90.4 & & & $3 X$ & Hortus Bulborum, Limmen & As T. agenensis $^{\mathrm{a}}$ \\
\hline 6.2 & T. aleppensis Boiss. ex Regel & 93.6 & 95.3 & 1.4 & $3 X$ & CPRO 84258 ex P Visser & \\
\hline $2 \lim 37$ & T. aleppensis Boiss. ex Regel & 94.3 & & & $3 X$ & Hortus Bulborum, Limmen & \\
\hline 6.1 & T. aleppensis Boiss. ex Regel & 94.8 & & & $3 X$ & Bulb Research Centre, Lisse & \\
\hline $\operatorname{sg} 1$ & T. aleppensis Boiss. ex Regel & 96.7 & & & $3 X$ & ex J. de Groot & \\
\hline $\operatorname{sg} 7$ & T. aleppensis Boiss. ex Regel & 97.3 & & & $3 X$ & ex J. de Groot & \\
\hline \multicolumn{8}{|c|}{ Section Tulipa } \\
\hline JPT1 & T. suaveolens Roth & 60.2 & 61.7 & 1.7 & $2 X$ & ex J. Tyssen & \\
\hline $\operatorname{cb} 63$ & T. suaveolens Roth & 60.8 & & & $2 X$ & ex C. Breed & As $T$. schrenkii hort. \\
\hline JPT2 & T. suaveolens Roth & 60.9 & & & $2 X$ & ex J. Tyssen & As $T$. schrenkii hort. \\
\hline 1620 & T. suaveolens Roth & 61.4 & & & $2 X$ & Hortus Bulborum, Limmen & As $T$. schrenkii hort. \\
\hline $\operatorname{cb} 34$ & T. suaveolens Roth & 62.9 & & & $2 X$ & ex C. Breed & 'Duc van Tol' \\
\hline w12 & T. suaveolens Roth & 64.2 & & & $2 X$ & ex Wageningen BG & \\
\hline $\mathrm{BZ}$ & T. schrenkii Regel & 60.3 & 61.5 & 1.3 & $2 X$ & Karalar plain, Crimea & ex J. Ruksans \\
\hline BZ07 & T. schrenkii Regel & 61.7 & & & $2 X$ & Korgalzhin, Tengislake, Kazak. & 2. Yellow flower \\
\hline BZ07 & T. schrenkii Regel & 61.7 & & & $2 X$ & Korgalzhin, Tengislake, Kazak. & 3. White flower \\
\hline BZ07 & T. schrenkii Regel & 61.8 & & & $2 X$ & Korgalzhin, Tengislake, Kazak. & 1. Pink flower \\
\hline 76.2 & T. schrenkii Regel & 62.1 & & & $2 X$ & Bulb Research Centre, Lisse & \\
\hline $\operatorname{sgl}$ & T. eichleri Regel & 62.7 & 62.7 & 1.8 & $2 X$ & W. of Quba, N. Azerbaijan & \\
\hline 78.1 & T. sosnovskyi Achv.\& Mirz. & 62.6 & 63.4 & 1.6 & $2 X$ & Bulb Research Centre, Lisse & \\
\hline $\lim 16$ & T. sosnovskyi Achv.\& Mirz. & 63.9 & & & $2 X$ & Hortus Bulborum, Limmen & \\
\hline D5314 & T. sosnovskyi Achv.\& Mirz. & 64.3 & & & $2 X$ & ex J. de Groot & \\
\hline $\operatorname{cb} 25$ & T. sosnovskyi Achv.\& Mirz. & 64.5 & & & $2 X$ & ex C. Breed & \\
\hline sgl & T. sosnovskyi Achv.\& Mirz. & 62.1 & & & $2 X$ & Meghri, Z. Armenia & \\
\hline $\operatorname{sgl}$ & T. sosnovskyi Achv.\& Mirz. & 62.9 & & & $2 X$ & Meghri, Z. Armenia & \\
\hline $\operatorname{sgl}$ & T. florenskyi Woronow & 64.2 & 64.2 & 0.8 & $2 X$ & Meghri, Z. Armenia & \\
\hline 45.1 & T. karabachensis Grossh. & 64.0 & 65.6 & 1.6 & $2 X$ & Caucasus & \\
\hline 45.2 & T. karabachensis Grossh. & 64.7 & & & $2 X$ & ex Letland & \\
\hline $\operatorname{cb} 72$ & T. karabachensis Grossh. & 65.6 & & & $2 X$ & ex C. Breed & \\
\hline $2 \lim 38$ & T. karabachensis Grossh. & 65.6 & & & $2 X$ & Hortus Bulborum, Limmen & \\
\hline $\operatorname{Lim} 20$ & T. karabachensis Grossh. & 65.8 & & & $2 X$ & Hortus Bulborum, Limmen & \\
\hline D5104 & T. karabachensis Grossh. & 67.9 & & & $2 X$ & ex J. de Groot & \\
\hline sgl & T. karabachensis Grossh. & 67.0 & & & $2 X$ & Meghri, Z. Armenia & As $T$. confusa ${ }^{\text {a }}$, pink flower \\
\hline $\operatorname{sgl}$ & T. karabachensis Grossh. & 66.1 & & & $2 X$ & Meghri, Z. Armenia & As $T$. confusa $a^{\text {a }}$, red flower \\
\hline $\operatorname{sgl}$ & T. karabachensis Grossh. & 65.5 & & & $2 X$ & Meghri, Z. Armenia & As $T$. confusa ${ }^{\text {a }}$, pink flower \\
\hline $\operatorname{sgl}$ & T. karabachensis Grossh. & 67.8 & & & $2 X$ & Meghri, Z. Armenia & As $T$. confusa ${ }^{\text {a }}$, pink flower \\
\hline 26.1 & T. karabachensis Grossh. & 65.6 & & & $2 X$ & Azerbaijan & \\
\hline 40.2 & T. hungarica Borbas & 64.7 & 65.6 & 2.3 & $2 X$ & CPRO 78392-2 & \\
\hline 40.1 & T. hungarica Borbas & 64.8 & & & $2 X$ & CPRO 78392-6, Budapest BG & \\
\hline $\lim 28$ & T. hungarica Borbas & 65.5 & & & $2 X$ & Hortus Bulborum, Limmen & \\
\hline
\end{tabular}


Table 1 continued

\begin{tabular}{|c|c|c|c|c|c|c|c|}
\hline \multicolumn{8}{|c|}{ Genus Tulipa L. (Liliaceae) } \\
\hline Coll. no. & & $\begin{array}{l}2 C \text { DNA } \\
(\mathrm{pg})\end{array}$ & Average & $\mathrm{SD}$ & Ploidy $^{b}$ & Origin & $\begin{array}{l}\text { Received as/ } \\
\text { cultivar }\end{array}$ \\
\hline \multirow[t]{2}{*}{40.3} & T. hungarica Borbas & 66.0 & & & $2 X$ & Bulb Research Centre, Lisse & \\
\hline & $\begin{array}{l}\text { T. hungarica } \text { var. } \\
\text { urumoffii } \text { Hayek }\end{array}$ & 65.7 & & & $2 X$ & ex commerce & As $T$. urumoffii ${ }^{\mathrm{a}}$ \\
\hline 91.1 & $\begin{array}{l}\text { T. hungarica var. urumoffii } \\
\text { Hayek }\end{array}$ & & & & $2 X$ & Budapest BG 75102-1 & As $T$. urumoffii $i^{\mathrm{a}}$ \\
\hline $\operatorname{cb} 26$ & T. rhodopaea (Velen.) Velen. & 67.6 & 68.6 & 2.1 & $2 X$ & Rhodope Mntns, Bulgaria & \\
\hline 72.1 & T. rhodopaea (Velen.) Velen. & 68.2 & & & $2 X$ & Rhodope Mntns, Bulgaria & \\
\hline 72.2 & T. rhodopaea (Velen.) Velen. & 68.5 & & & $2 X$ & Bulb Research Centre, Lisse & \\
\hline $\lim 14$ & T. rhodopaea (Velen.) Velen. & 70.0 & & & $2 X$ & Rhodope Mntns, Bulgaria & \\
\hline $\operatorname{sg} 309$ & T. gesneriana? L. & 68.6 & 69.2 & 1.2 & $2 X$ & Kordestan SW Negel, Iran & Seed JJA02 \\
\hline $\operatorname{sg} 233$ & T. gesneriana? L. & 69.0 & & & $2 X$ & Macedonia, ex BG Got. & as $\boldsymbol{T}$. scardica ${ }^{\mathrm{a}}$ \\
\hline $\operatorname{cb} 40$ & T. gesneriana? L. & 69.9 & & & $2 X$ & $\begin{array}{l}\text { N Cauc., Piatogorsk, } \\
\text { Krasnodar }\end{array}$ & as T. schrenkii? \\
\hline cb18 & T. gesneriana L. & 67.3 & 68.7 & 2.0 & $2 X$ & ex C. Breed & As $T$. viridiflor $a^{\mathrm{a}}$ \\
\hline 30.1 & T. gesneriana $\mathrm{L}$. & 67.9 & & & $2 X$ & Bulb Research Centre, Lisse & As $\boldsymbol{T}$. didieri ${ }^{\mathrm{a}}$ \\
\hline 2.1 & T. gesneriana $\mathrm{L}$. & 67.7 & & & $2 X$ & Bulb Research Centre, Lisse & As $\boldsymbol{T}$. acuminata $^{\mathrm{a}}$ \\
\hline $\operatorname{cb} 17$ & T. gesneriana $\mathrm{L}$. & 68.2 & & & $2 X$ & ex C. Breed & As $\boldsymbol{T} \cdot$ fulgens $^{\mathrm{a}}$ \\
\hline $\operatorname{cb} 21$ & T. gesneriana $\mathrm{L}$. & 68.2 & & & $2 X$ & ex C. Breed & As $\boldsymbol{T}$. marjolettii ${ }^{\mathrm{a}}$ \\
\hline $2 \lim 27$ & T. gesneriana $\mathrm{L}$. & 67.3 & & & $2 X$ & Hortus Bulborum, Limmen & As $T$. marjolettii ${ }^{\mathrm{a}}$ \\
\hline 66.1 & T. gesneriana $\mathrm{L}$. & 68.7 & & & $2 X$ & Bulb Research Centre, Lisse & As $T \cdot$ platystigma ${ }^{\mathrm{a}}$ \\
\hline 54.1 & T. gesneriana L. & 68.8 & & & $2 X$ & Bulb Research Centre, Lisse & $\begin{array}{l}\text { As } T \text {. mauritiana }{ }^{\mathrm{a}} \\
\text { 'Cindy' }\end{array}$ \\
\hline $\operatorname{cb} 24$ & T. gesneriana $\mathrm{L}$. & 69.3 & & & $2 X$ & ex C. Breed & As $T$. aximensis $^{\mathrm{a}}$ \\
\hline 95 & T. gesneriana L. & 69.4 & & & $2 X$ & Bulb Research Centre, Lisse & As $T$. saracenica ${ }^{a}$ \\
\hline $\operatorname{cb} 22$ & T. gesneriana $\mathrm{L}$. & 70.1 & & & $2 X$ & ex C. Breed & As $T$. didieri ${ }^{\mathrm{a}}$ \\
\hline $\operatorname{cbd} 27$ & T. gesneriana $\mathrm{L}$. & 70.1 & & & $2 X$ & ex C. Breed & As $T \cdot$ passeriniana $a^{\mathrm{a}}$ \\
\hline 34.1 & T. gesneriana L. & 68.8 & & & $2 X$ & red flower, Grengiols, Suisse & As $T$. grengiolensis $^{\mathrm{a}}$ \\
\hline $\operatorname{cb} 23$ & T. gesneriana $\mathrm{L}$. & 70.4 & & & $2 X$ & ex C. Breed & As $T$. grengiolensis $^{\mathrm{a}}$ \\
\hline $\mathrm{TC}$ & T. gesneriana $\mathrm{L}$. & 67.7 & & & $2 X$ & Yellow fl., Grengiols, Suisse & As $\boldsymbol{T}$. grengiolensis $^{\mathrm{a}}$ \\
\hline 36.2 & T. gesneriana $\mathrm{L}$. & 67.0 & & & $2 X$ & CPRO 84266, ex W. Kooiman & As $\boldsymbol{T}$. galatica ${ }^{\mathrm{a}}$ Hort. \\
\hline $\operatorname{cb} 47$ & T. gesneriana $\mathrm{L}$. & 68.1 & & & $2 X$ & ex P. Nyssen & As $\boldsymbol{T}$. galatica ${ }^{\mathrm{a}}$ Hort. \\
\hline $\operatorname{sg} 232$ & T. gesneriana $\mathrm{L}$. & 68.9 & & & $2 X$ & ex J. de Groot & As $\boldsymbol{T}$. galatica ${ }^{\mathrm{a}}$ Hort. \\
\hline 36.1 & T. gesneriana $\mathrm{L}$. & 69.0 & & & $2 X$ & Bulb Research Centre, Lisse & As $\boldsymbol{T}$. galatica ${ }^{\mathrm{a}}$ Hort. \\
\hline $\lim 25$ & T. gesneriana $\mathrm{L}$. & 67.7 & & & $2 X$ & Hortus Bulborum, Limmen & As $\boldsymbol{T}$. galatica ${ }^{\mathrm{a}}$ Hort. \\
\hline Lieser & T. gesneriana L. & 70.2 & & & $2 X$ & L. Lieser France, bulblet & As $\boldsymbol{T}$. rubidusa ${ }^{\mathrm{a}}$ Lieser \\
\hline Lieser & T. gesneriana $\mathrm{L}$. & 70.5 & & & $2 X$ & L. Lieser France, bulblet & As $\boldsymbol{T}$. sedunii ${ }^{\mathrm{a}}$ Lieser \\
\hline \multicolumn{8}{|c|}{ Subgenus Eriostemons (Boiss.) Raamsd. } \\
\hline \multicolumn{8}{|c|}{ Section Sylvestres (Baker) Baker } \\
\hline D5706 & T. patens C.Agarth ex Schult. & 54.2 & 55.0 & 1.3 & $2 X$ & Altai, Kazakhstan & \\
\hline $\operatorname{sg} 201$ & T. patens C.Agarth ex Schult. & 54.5 & & & $2 X$ & Kamenogorsk & \\
\hline BZ07 & T. patens C.Agarth ex Schult. & 54.8 & & & $2 X$ & Tengis lake, Kazakhstan no 3 & \\
\hline BZ07 & T. patens C.Agarth ex Schult. & 55.4 & & & $2 X$ & Tengis lake, Kazakhstan no 1 & \\
\hline sg & T. patens C.Agarth ex Schult. & 55.4 & & & $2 X$ & Kamenogorsk & \\
\hline BZ07 & T. patens C.Agarth ex Schult. & 55.5 & & & $2 X$ & Tengis lake, Kazakhstan no 2 & \\
\hline 18.1 & T. biebersteiniana Schult. f & 56.1 & 56.7 & 1.4 & $2 X$ & CPRO 73108, ex Stavropol BG & \\
\hline cb51 & T. biebersteiniana Schult. f & 56.5 & & & $2 X$ & ex C. Breed & \\
\hline 10.2 & T. biebersteiniana Schult. f & 56.7 & & & $2 X$ & France & As $T$. australis \\
\hline
\end{tabular}


Table 1 continued

\begin{tabular}{|c|c|c|c|c|c|c|c|}
\hline \multicolumn{8}{|c|}{ Genus Tulipa L. (Liliaceae) } \\
\hline Coll. no. & & $\begin{array}{l}2 C \text { DNA } \\
(\mathrm{pg})\end{array}$ & Average & $\mathrm{SD}$ & Ploidy $^{\mathrm{b}}$ & Origin & $\begin{array}{l}\text { Received as/ } \\
\text { cultivar }\end{array}$ \\
\hline CB65 & T. biebersteiniana Schult. f & 56.7 & & & $2 X$ & ex C. Breed & \\
\hline D 5022 & T. biebersteiniana Schult. f & 57.0 & & & $2 X$ & ex J. de Groot & \\
\hline 82.3 & T. biebersteiniana Schult. f & 57.1 & & & $2 X$ & $\begin{array}{l}\text { Bulb Research Centre, } \\
\text { Lisse }\end{array}$ & As T. sylvestris \\
\hline 102.1 & T. biebersteiniana Schult. f & 57.9 & & & $2 X$ & Peloponesus, Greece & As $T$. australis \\
\hline D5705a & T. biebersteiniana Schult. f & 56.0 & & & $2 X$ & Karalar Steppe & As T. ophiophylla ${ }^{a}$ \\
\hline $\operatorname{cb} 35$ & T. hageri Heldreich & 57.7 & 57.7 & 0.7 & $2 X$ & ex C. Breed & ‘Splendens’ \\
\hline 37.3 & T. hageri Heldreich & 85.9 & 85.9 & 1.1 & $3 X$ & Large form & \\
\hline $\operatorname{cb} 37$ & T. orphanidea Boiss. ex Heldr. & 59.0 & 59.6 & 1.3 & $2 X$ & ex C. Breed & As T. sprengeri Hort. \\
\hline $\operatorname{cb} 38$ & T. orphanidea Boiss. ex Heldr. & 59.1 & & & $2 X$ & ex C. Breed & \\
\hline 64.4 & T. orphanidea Boiss. ex Heldr. & 59.4 & & & $2 X$ & $\begin{array}{l}\text { Bulb Research Centre, } \\
\text { Lisse }\end{array}$ & $\begin{array}{l}\text { As } T \text {. orphanidea } \\
\text { 'Flava' }\end{array}$ \\
\hline testc. & T. orphanidea Boiss. ex Heldr. & 59.4 & & & $2 X$ & $\begin{array}{l}\text { Bulb Research Centre, } \\
\text { Lisse }\end{array}$ & As $T \cdot$ goulimyi $^{\mathrm{a}}$ \\
\hline D5711 & T. orphanidea Boiss. ex Heldr. & 59.9 & & & $2 X$ & BG Gotenburg & As $T$. goulimyi ${ }^{\text {a }}$ \\
\hline Aanesen & T. orphanidea Boiss. ex Heldr. & 60.7 & & & $2 X$ & Kythira, Greece & As $\boldsymbol{T}$. goulimyi ${ }^{\text {a }}$ \\
\hline 64.2 & T. orphanidea Boiss. ex Heldr. & 88.5 & 88.7 & 1.5 & $3 X$ & CPRO 95192 & \\
\hline D5713 & T. orphanidea Boiss. ex Heldr. & 88.4 & & & $3 X$ & Lesbos, Greece & As T. theophrasti ${ }^{\text {a }}$ \\
\hline Aanesen & $\begin{array}{l}\text { T. orphanidea ssp. doerfleri (Gand.) } \\
\text { Zonn. }\end{array}$ & 86.6 & & & $3 X$ & Gious-Kambos, Crete & As $T$. doerfleri ${ }^{\mathrm{a}}$ \\
\hline 29.2 & $\begin{array}{l}\text { T. orphanidea ssp. doerfleri (Gand.) } \\
\text { Zonn. }\end{array}$ & 87.2 & & & $3 X$ & Mt .Kedros, Spili, Crete & As $T$. doerfleri $^{\mathrm{a}}$ \\
\hline Aanesen & $\begin{array}{l}\text { T. orphanidea ssp. doerfleri (Gand.) } \\
\text { Zonn. }\end{array}$ & 87.9 & & & $3 X$ & Spili, Crete & As $T$. doerfleri $^{\mathrm{a}}$ \\
\hline 29.1 & $\begin{array}{l}\text { T. orphanidea ssp. doerfleri (Gand.) } \\
\text { Zonn. }\end{array}$ & 89.1 & & & $3 X$ & Spili, Crete & As $T$. doerfleri $^{\mathrm{a}}$ \\
\hline $\operatorname{cb} 62$ & $\begin{array}{l}\text { T. orphanidea ssp. doerfleri (Gand.) } \\
\text { Zonn. }\end{array}$ & 93.2 & & & $3 X$ & Spili, Crete & As $T$. doerfleri $^{\mathrm{a}}$ \\
\hline 64.3 & $\begin{array}{l}\text { T. orphanidea ssp. whittallii Boiss. ex } \\
\text { Heldr. }\end{array}$ & 115.1 & 115.9 & 3.2 & $4 X$ & CPRO 89109 & As $T$. whittallii ${ }^{\text {a }}$ \\
\hline 94.1 & $\begin{array}{l}\text { T. orphanidea ssp. whittallii Boiss. ex } \\
\text { Heldr. }\end{array}$ & 116.6 & & & $4 X$ & $\begin{array}{l}\text { Bulb Research Centre, } \\
\text { Lisse }\end{array}$ & As $T$. whittallii ${ }^{\text {a }}$ \\
\hline 10.1 & T. sylvestris ssp. australis (Link) Pamp. & 61.2 & 62.0 & 1.5 & $2 X$ & Andorra & \\
\hline 10.4 & T. sylvestris ssp. australis (Link) Pamp. & 61.9 & & & $2 X$ & CPRO 89103 & \\
\hline 10.5 & T. sylvestris ssp. australis (Link) Pamp. & 62.8 & & & $2 X$ & Ronda, Spain & \\
\hline Aanesen & T. sylvestris ssp. sylvestris L. & 89.8 & 90.4 & 1.0 & $3 X$ & Mandal, Norway & \\
\hline Aanesen & T. sylvestris ssp. sylvestris L. & 89.9 & & & $3 X$ & Arendal, Norway & \\
\hline Aanesen & T. sylvestris ssp. sylvestris $\mathrm{L}$. & 91.4 & & & $3 X$ & Little Torungen, Norway & \\
\hline $\mathrm{cb} 41$ & T. sylvestris ssp. sylvestris L. & 118.6 & 120.9 & 3.2 & $4 X$ & Rich flowering & \\
\hline D 5133 & T. sylvestris ssp. sylvestris $\mathrm{L}$. & 119.0 & & & $4 X$ & V.S.03. & \\
\hline 82.1 & T. sylvestris $\mathrm{ssp}$. sylvestris $\mathrm{L}$. & 119.4 & & & $4 X$ & CPRO 73143 & \\
\hline 82.4 & T. sylvestris ssp. sylvestris L. & 120.7 & & & $4 X$ & Tatra Mntms & T. sylvestris 'Tatrensis' \\
\hline 82.5 & T. sylvestris ssp. sylvestris L. & 121.0 & & & $4 X$ & CPRO & \\
\hline BGL & T. sylvestris ssp. sylvestris L. & 122.8 & & & & Bot. Garden, Leiden & \\
\hline 82.2 & T. sylvestris ssp. sylvestris L. & 122.8 & & & $4 X$ & CPRO & As $T$. australis \\
\hline D 5133a & T. sylvestris ssp. sylvestris L. & 123.0 & & & $4 X$ & Tabriz, Iran (V.S. 04) & \\
\hline $\mathrm{cb} 46$ & T. sprengeri Baker & 63.7 & 64.6 & 1.8 & $2 X$ & ex C. Breed & \\
\hline 79.1 & T. sprengeri Baker & 64.5 & & & $2 X$ & $\begin{array}{l}\text { Bulb Research Centre, } \\
\text { Lisse }\end{array}$ & \\
\hline
\end{tabular}


Table 1 continued

\begin{tabular}{|c|c|c|c|c|c|c|c|}
\hline \multicolumn{8}{|c|}{ Genus Tulipa L. (Liliaceae) } \\
\hline \multicolumn{2}{|l|}{ Coll. no. } & \multirow{2}{*}{$\begin{array}{l}\begin{array}{l}2 \boldsymbol{C} \text { DNA } \\
(\mathrm{pg})\end{array} \\
65.5\end{array}$} & \multirow[t]{2}{*}{ Average } & \multirow[t]{2}{*}{$\mathrm{SD}$} & \multirow{2}{*}{$\begin{array}{l}\text { Ploidy }^{\mathrm{b}} \\
2 X\end{array}$} & \multirow{2}{*}{$\begin{array}{l}\text { Origin } \\
\text { Bulb Research Centre, Lisse }\end{array}$} & \multirow[t]{2}{*}{$\begin{array}{l}\text { Received as/ } \\
\text { cultivar }\end{array}$} \\
\hline Z026 & T. sprengeri Baker & & & & & & \\
\hline 70.1 & T. primulina Baker & 63.7 & 64.2 & 1.9 & $2 X$ & Bulb Research Centre, Lisse & \\
\hline $2 \lim 41$ & T. primulina Baker & 63.7 & & & $2 X$ & Hortus Bulborum, Limmen & \\
\hline CB64 & T. primulina Baker & 64.4 & & & $2 X$ & ex Monocot nursery & \\
\hline $\operatorname{cb} 71$ & T. primulina Baker & 65.2 & & & $2 X$ & ex C. Breed & \\
\hline 10.3 & T. celsiana $\mathrm{DC}$ & 65.6 & 66.0 & 0.4 & $2 X$ & CPRO 75106 & As $T$. australis \\
\hline BZ01 & T. celsiana $\mathrm{DC}$ & 65.9 & & & $2 X$ & Katara Pass, Greece & As $T$. australis \\
\hline $\operatorname{cb} 43$ & T. celsiana $\mathrm{DC}$ & 66.2 & & & $2 X$ & ex C. Breed & \\
\hline 70.2 & T. celsiana $\mathrm{DC}$ & 66.3 & & & $2 X$ & CPRO 69308, Dar-UlBaider, Leb. & As $T$. primulina \\
\hline \multicolumn{8}{|c|}{ Section Biflores A.D.Hall ex Zonn. \& Veldk. } \\
\hline $\mathrm{sg} 9 / 5$ & $T$. sp. & 48.0 & 48.0 & 0.4 & $2 X$ & Dzjungaric Ala-Tau & \\
\hline $\operatorname{cb} 81$ & T. tarda Stapf & 50.2 & 51.5 & 1.3 & $2 X$ & ex van Tubergen & \\
\hline \multirow[t]{2}{*}{$\operatorname{cb} 45$} & T. tarda Stapf & 50.7 & & & $2 X$ & ex C. Breed & As $T$. urumiensis $^{\mathrm{a}}$ \\
\hline & T. tarda Stapf & 50.9 & & & $2 X$ & ex commerce & As $T$. urumiensis $^{\mathrm{a}}$ \\
\hline 83.2 & T. $\operatorname{tarda}$ Stapf & 51.2 & & & $2 X$ & Almaty, Kazakhstan & \\
\hline $\operatorname{cb} 82$ & T. tarda Stapf & 51.5 & & & $2 X$ & ex C. Breed & \\
\hline 92.2 & T. tarda Stapf & 51.5 & & & $2 X$ & Bulb Research Centre, Lisse & As $T$. urumiensis $^{\mathrm{a}}$ \\
\hline $\operatorname{Lim} 22$ & T. $\operatorname{tarda}$ Stapf & 51.9 & & & $2 X$ & Hortus Bulborum, Limmen & As T. urumiensis ${ }^{\text {a }}$ 'Tity Star' \\
\hline \multirow[t]{2}{*}{ sg’08 } & T. $\operatorname{tarda}$ Stapf aff. & 51.9 & & & $2 X$ & Kyrgyzstan & As $T$. sp. \\
\hline & T. tarda Stapf & 52.0 & & & $2 X$ & Bulb Research Centre, Lisse & As hybrid? \\
\hline 83.1 & T. $\operatorname{tarda}$ Stapf & 52.0 & & & $2 X$ & Bulb Research Centre, Lisse & \\
\hline 83.3 & T. tarda Stapf & 52.1 & & & $2 X$ & Bulb Research Centre, Lisse & Dark form \\
\hline 92.1 & T. tarda Stapf & 52.5 & & & $2 X$ & CPRO 64177 & As $T$. urumiensis ${ }^{\mathrm{a}}$ \\
\hline D5900 & T. turcomanica B. Fdtsch. & 76.3 & 76.3 & 1.2 & $3 X$ & $\begin{array}{l}\text { Foothills Kopet Dagh, } \\
\text { Turkmenistan }\end{array}$ & \\
\hline $\operatorname{sg} 40$ & T. dasystemon Regel & 51.2 & 51.5 & 1.3 & $2 X$ & Coll. seed: 44 & \\
\hline 28.2 & T. dasystemon Regel & 51.4 & & & $2 X$ & CPRO 97263 & \\
\hline D $5017 \mathrm{~b}$ & T. dasystemon Regel & 53.3 & & & $2 X$ & Kugart valley, Kyrgyzstan & \\
\hline sgl & T. dasystemon Regel & 50.0 & & & $2 X$ & Kyrgyzstan & \\
\hline $\operatorname{sgl}$ & T. dasystemon Regel & 99.6 & 99.6 & 1.2 & $4 X$ & Kyrgyzstan & As $T$. dasystemonoides \\
\hline 103.1 & T. regelii Elwes. & 52.4 & 52.4 & 1.5 & $2 X$ & Tamgalitas, Kazakhstan & \\
\hline $\operatorname{sg}$ & $T$. sp. nov. & 51.9 & 52.4 & 0.4 & $2 X$ & Altai, Kazakhstan & As $T$. biflora \\
\hline 4.3 & T. sp. nov. & 52.3 & & & $2 X$ & Altai, Kazakhstan & As $T$. biflora \\
\hline D5500 & T. sp. nov. & 52.6 & & & $2 X$ & Altai, Kazakhstan 2005 & As $T$. biflora \\
\hline \multirow[t]{3}{*}{ D5901b } & T. sp. nov. & 52.9 & & & $2 X$ & Altai, Kazakhstan 2006 & As $T$. biflora \\
\hline & T. neustruevae Pobed. & 53.9 & 54.8 & 0.7 & $2 X$ & ex Commerce & As $T$. dasystemon \\
\hline & T. neustruevae Pobed. & 54.2 & & & $2 X$ & ex Commerce & As $T$. dasystemon \\
\hline 28.1 & T. neustruevae Pobed. & 54.2 & & & $2 X$ & Bulb Research Centre, Lisse & As $T$. dasystemonoides? \\
\hline $\operatorname{cb} 83$ & T. neustruevae Pobed. & 54.4 & & & $2 X$ & ex C. Breed & As $T$. dasystemon \\
\hline D 5018 & T. neustruevae Pobed. & 54.7 & & & $2 X$ & ex J. de Groot & \\
\hline 59.1 & T. neustruevae Pobed. & 55.0 & & & $2 X$ & Bulb Research Centre, Lisse & \\
\hline $\operatorname{cb} 84$ & T. neustruevae Pobed. & 55.4 & & & $2 X$ & ex C. Breed & \\
\hline D 5018a & T. neustruevae Pobed. & 55.6 & & & $2 X$ & ex J. de Groot & \\
\hline D 5017 & T. neustruevae Pobed. & 56.0 & & & $2 X$ & ex J. de Groot & As $T$. dasystemon \\
\hline $\operatorname{cb} 42$ & T. biflora Pall. & 53.4 & 55.7 & 1.8 & $2 X$ & ex C. Breed & \\
\hline 20.2 & T. biflora Pall. & 54.4 & & & $2 X$ & Bulb Research Centre, Lisse & 'Yellow Form' \\
\hline
\end{tabular}


Table 1 continued

\begin{tabular}{|c|c|c|c|c|c|c|c|}
\hline \multicolumn{8}{|c|}{ Genus Tulipa L. (Liliaceae) } \\
\hline \multicolumn{2}{|l|}{ Coll. no. } & \multirow{2}{*}{$\begin{array}{l}\begin{array}{l}2 C \text { DNA } \\
(\mathrm{pg})\end{array} \\
54.5\end{array}$} & \multirow[t]{2}{*}{ Average } & \multirow[t]{2}{*}{ SD } & \multirow{2}{*}{$\begin{array}{l}\text { Ploidy }^{\mathrm{b}} \\
2 X\end{array}$} & \multirow{2}{*}{$\begin{array}{l}\text { Origin } \\
\text { ex J. Ruksans }\end{array}$} & \multirow{2}{*}{$\begin{array}{l}\begin{array}{l}\text { Received as/ } \\
\text { cultivar }\end{array} \\
\text { 'Starlight' }\end{array}$} \\
\hline D 5013 & T. biflora Pall. & & & & & & \\
\hline D 5134 & T. biflora Pall. & 55.2 & & & $2 X$ & Kara-Tau Mntns & As $T$. orthopoda \\
\hline $\operatorname{cb} 59$ & T. biflora Pall. & 55.6 & & & $2 X$ & ex C. Breed & As $\boldsymbol{T}$. binutans ${ }^{\mathrm{a}}$ \\
\hline 20.1 & T. biflora Pall. & 56.1 & & & $2 X$ & Bulb Research Centre, Lisse & \\
\hline D 5014 & T. biflora Pall. & 56.2 & & & $2 X$ & ex J. de Groot & \\
\hline BZ07 & T. biflora Pall. & 56.3 & & & $2 X$ & Kabchagal, Kazakhstan & As $T$. talievii ${ }^{\mathrm{a}}$ \\
\hline D5914 & T. biflora Pall. & 57.3 & & & $2 X$ & Kyzyl-Kul, Kazakhstan & As T. busheana $a^{a}$ \\
\hline D5914a & T. biflora Pall. & 57.6 & & & $2 X$ & Kabchagal, Kazakhstan & As T. talievii ${ }^{\mathrm{a}}$ \\
\hline $\operatorname{cb} 32$ & T. biflora Pall. & 109.1 & 110.0 & & $4 X$ & ex C. Breed & \\
\hline D 5011f & T. biflora Pall. & 110.8 & & & $4 X$ & Chimgan, Kazakhstan & \\
\hline D 5012 & T. turkestanica Regel & 55.7 & 56.2 & 0.5 & $2 X$ & Aman-Kutan, Uzbekistan & Coll. J. Ruksans \\
\hline $\mathrm{cb} 67 / \mathrm{Tc}$ & T. turkestanica Regel & 56.3 & & & $2 X$ & Dshizak, Nuratau mnts & Coll. J. Ruksans \\
\hline $\operatorname{cb} 55$ & T. turkestanica Regel & 56.6 & & & $2 X$ & Cambridge BG & As T. bifloriformis ${ }^{\mathrm{a}}$ \\
\hline sg & T. turkestanica Regel & 112.4 & 113.4 & & $4 X$ & ex J. de Groot & Sprout brown, hairy \\
\hline sg & T. turkestanica Regel & 114.3 & & & $4 X$ & ex J. de Groot & Sprout brown, hairy \\
\hline D5901b & T. polychroma Stapf & 54.5 & 56.7 & 2.0 & $2 X$ & Yugoslavia, CPRO 79103 & As $T$. mariannae $e^{a}$ \\
\hline sgl & T. polychroma Stapf & 55.3 & & & $2 X$ & Vayak, M2, Armenia & \\
\hline $\operatorname{cb} 30$ & T. polychroma Stapf & 55.6 & & & $2 X$ & ex C. Breed & \\
\hline 67.1 & T. polychroma Stapf & 57.5 & & & $2 X$ & ex C. Breed & \\
\hline 67.4 & T. polychroma Stapf & 58.7 & & & $2 X$ & CPRO 91106 & \\
\hline sg & T. polychroma Stapf & 58.8 & & & $2 X$ & RRW00123 & As $T$. biflora \\
\hline 67.3 & T. polychroma Stapf & 84.4 & 84.4 & 2.1 & $3 X$ & CPRO 95201 & \\
\hline 77.2 & T. sogdiana Bunge & 57.2 & 57.4 & 1.4 & $2 X$ & CPRO 95200 & \\
\hline w08 & T. sogdiana Bunge & 57.5 & & & $2 X$ & ex Wageningen BG & \\
\hline $\operatorname{sg} 39$ & T. dasystemoniodes Vved. & 57.3 & 57.7 & 1.3 & $2 X$ & Kyrgyzstan & sp. 43, flower white \\
\hline D 5013a & T. dasystemoniodes Vved. & 57.7 & & & $2 X$ & Zaamin Reserve, Uzbekistan & \\
\hline D 5111 & T. dasystemoniodes Vved. & 58.0 & & & $2 X$ & ex J. Ruksans $02 . / 03$ & \\
\hline D 5088 & T. orithyioides Vved. & 58.3 & 58.3 & 1.7 & $2 X$ & ex J. de Groot & \\
\hline D 5086a & T. orithyioides Vved. & 87.1 & 87.1 & & $3 X$ & Tajikistan & \\
\hline $\operatorname{sg} 28$ & T. orthopoda Vved. & 58.5 & 59.3 & 1.4 & $2 X$ & Kujuk Pass, Kazakhstan & \\
\hline BZ07 & T. orthopoda Vved. & 60.1 & & & $2 X$ & Kujuk Pass, Kazakhstan & \\
\hline D5905 & T. binutans Vved. & 59.3 & 59.4 & 1.5 & $2 X$ & Muyun Kum desert, Kazakhstan & \\
\hline BZ07 & T. binutans Vved. & 59.4 & & & $2 X$ & Dzabagly, Kazakhstan & \\
\hline \multicolumn{8}{|c|}{ Section Saxatiles (Baker) Baker } \\
\hline $\operatorname{cb} 74$ & T. cretica Boiss. \& Heldr. & 57.9 & 60.5 & 2.2 & $2 X$ & ex C. Breed & \\
\hline 25.2 & T. cretica Boiss. \& Heldr. & 60.8 & & & $2 X$ & Ida Plateau, Crete & \\
\hline 25.1 & T. cretica Boiss. \& Heldr. & 61.6 & & & $2 X$ & CPRO76122 & \\
\hline 25.3 & T. cretica Boiss. \& Heldr. & 61.7 & & & $2 X$ & Bulb Research Centre, Lisse & \\
\hline 74.2 & T. saxatilis ssp. bakeri (Hall) Zonn. & 60.7 & 62.7 & 1.3 & $2 X$ & White Mntsn, Crete & \\
\hline Aanesen & T. saxatilis ssp. bakeri (Hall) Zonn. & 62.1 & & & $2 X$ & W. Omalos plateau, Crete, cult land & As $\boldsymbol{T}$. bakeri $^{\mathrm{a}}$ \\
\hline 17.1 & T. saxatilis ssp. bakeri (Hall) Zonn. & 62.3 & & & $2 X$ & Bulb Research Centre, Lisse & 'Lilac Wonder' \\
\hline Aanesen & T. saxatilis ssp. bakeri (Hall) Zonn. & 62.9 & & & $2 X$ & Gious-Kambos, Spili, Crete & \\
\hline $\operatorname{cb} 48$ & T. saxatilis $\mathrm{ssp}$. bakeri (Hall) Zonn. & 63.2 & & & $2 X$ & ex M. Salmon & As $\boldsymbol{T}$. bakeri $^{\text {a }}$ \\
\hline
\end{tabular}


Table 1 continued

\begin{tabular}{|c|c|c|c|c|c|c|c|}
\hline \multicolumn{8}{|c|}{ Genus Tulipa L. (Liliaceae) } \\
\hline \multicolumn{2}{|l|}{ Coll. no. } & \multirow{2}{*}{$\begin{array}{l}2 C \text { DNA } \\
(\mathrm{pg})\end{array}$} & \multirow[t]{2}{*}{ Average } & \multirow{2}{*}{ SD } & \multirow{2}{*}{$\begin{array}{l}\text { Ploidy }^{\mathrm{b}} \\
2 X\end{array}$} & \multirow{2}{*}{$\begin{array}{l}\text { Origin } \\
\text { Bulb Research Centre, Lisse }\end{array}$} & \multirow{2}{*}{$\begin{array}{l}\begin{array}{l}\text { Received as/ } \\
\text { cultivar }\end{array} \\
\begin{array}{r}\text { 'Little Beauty' } \\
(\times \text { aucher.? })\end{array}\end{array}$} \\
\hline 13.1 & T. saxatilis ssp. bakeri (Hall) Zonn. & & & & & & \\
\hline Aanesen & T. saxatilis ssp. bakeri (Hall) Zonn. & 63.9 & & & $2 X$ & $\begin{array}{l}\text { W.Omalos plateau, Crete, } \\
\text { rocks }\end{array}$ & As $T$. bakeri $^{\mathrm{a}}$ \\
\hline 74.1 & T. saxatilis Sieber ex Spreng. & 92.8 & 93.1 & 1.7 & $3 X$ & Bulb Research Centre, Lisse & \\
\hline cb49 & T. saxatilis Sieber ex Spreng. & 93.4 & & & $3 X$ & ex M. Salmon & \\
\hline 71.11 & T. pulchella (Fenz ex Regel) Baker & 60.3 & 61.7 & 0.7 & $2 X$ & Bulb Research Centre, Lisse & Type 11 \\
\hline 71.1 & T. pulchella (Fenz ex Regel) Baker & 60.4 & & & $2 X$ & Bulb Research Centre, Lisse & Type 7 \\
\hline 71.7 & T. pulchella (Fenz ex Regel) Baker & 61.2 & & & $2 X$ & Bulb Research Centre, Lisse & Rose $48 \mathrm{a}$ \\
\hline 71.17 & T. pulchella (Fenz ex Regel) Baker & 61.4 & & & $2 X$ & Bulb Research Centre, Lisse & 'Liliput' \\
\hline 71.19 & T. pulchella (Fenz ex Regel) Baker & 61.7 & & & $2 X$ & Bulb Research Centre, Lisse & 'Pegasus' (wild coll.) \\
\hline 71.14 & T. pulchella (Fenz ex Regel) Baker & 61.7 & & & $2 X$ & Bulb Research Centre, Lisse & Type 14 \\
\hline 49.3 & T. pulchella (Fenz ex Regel) Baker & 61.8 & & & $2 X$ & Bulb Research Centre, Lisse & As $\boldsymbol{T}$. kurdica $^{\mathrm{a}}$ \\
\hline 71.13 & T. pulchella (Fenz ex Regel) Baker & 61.8 & & & $2 X$ & Bulb Research Centre, Lisse & Red form \\
\hline 71.5 & T. pulchella (Fenz ex Regel) Baker & 62.1 & & & $2 X$ & Bulb Research Centre, Lisse & Type 5 \\
\hline 11.1 & T. pulchella (Fenz ex Regel) Baker & 62.2 & & & $2 X$ & Bulb Research Centre, Lisse & 'Red Cup' \\
\hline 49.4 & T. pulchella (Fenz ex Regel) Baker & 62.2 & & & $2 X$ & IPTS & As $\boldsymbol{T}$. kurdica $^{\mathrm{a}}$ \\
\hline 71.2 & T. pulchella (Fenz ex Regel) Baker & 62.6 & & & $2 X$ & Bulb Research Centre, Lisse & 'Zephyr' (wild coll.) \\
\hline 49.1 & T. pulchella (Fenz ex Regel) Baker & 62.8 & & & $2 X$ & no 60 & As $\boldsymbol{T}$. kurdica $^{\mathrm{a}}$ \\
\hline 71.3 & T. humilis Herb. & 64.0 & 65.0 & 0.7 & $2 X$ & Sel. J. de Winter & As $\boldsymbol{T} \cdot$ pulchella $^{\mathrm{a}}$ \\
\hline 71.15 & T. humilis Herb. & 64.0 & & & $2 X$ & Bulb Research Centre, Lisse & $\begin{array}{l}\text { 'Albocoerulea- } \\
\text { oculata' }\end{array}$ \\
\hline $\operatorname{cb} 52$ & T. humilis Herb. & 64.4 & & & $2 X$ & ex C. Breed & As $\boldsymbol{T}$. aucheriana $\boldsymbol{a}^{\mathrm{a}}$ \\
\hline 9.1 & T. humilis Herb. & 64.6 & & & $2 X$ & Bulb Research Centre, Lisse & As $\boldsymbol{T}$. aucheriana ${ }^{\mathrm{a}}$ \\
\hline 71.2 & T. humilis Herb. & 64.8 & & & $2 X$ & Bulb Research Centre, Lisse & ‘Magenta Queen' \\
\hline 71.16 & T. humilis Herb. & 65.2 & & & $2 X$ & Bulb Research Centre, Lisse & 'Eastern Star' \\
\hline 38.2 & T. humilis Herb. & 65.4 & & & $2 X$ & Bulb Research Centre, Lisse & As $T \cdot$ pulchella \\
\hline 71.22 & T. humilis Herb. & 65.7 & & & $2 X$ & Bulb Research Centre, Lisse & As $T$. violacea hort. \\
\hline 71.18 & T. humilis Herb. & 65.8 & & & $2 X$ & Bulb Research Centre, Lisse & 'Odalisque' \\
\hline 71.21 & T. humilis Herb. & 66.0 & & & $2 X$ & Bulb Research Centre, Lisse & 'Persian Pearl' \\
\hline \multicolumn{8}{|c|}{ Genus Amana Honda } \\
\hline $\operatorname{sg} 50$ & A. edulis (Miq.) Honda & 114.7 & 114.7 & 4.3 & $4 X$ & ex G. Knoche & \\
\hline D 5057 & $\begin{array}{l}\text { A. erythronioides? (Baker) Tan \& } \\
\text { Hong }\end{array}$ & 50.7 & 50.7 & 3.0 & $2 X$ & Hythe Alpines 1999 & As T. edulis \\
\hline
\end{tabular}

\footnotetext{
a Synonym

b Ploidy was based on published chromosome numbers and here determined genome sizes
}

people accept three sections here: Biflores A.D. Hall ex Zonn. and Veldk., Sylvestres (Baker) Baker (syn.: Australes) and Saxatiles (Baker) Baker. Botschantzeva (1962), based mainly on Vvedenskii (1935), divided the tulips into six sections and section Tulipa (as Leiostemones) into eight groups that only partially coincide with the sections and series of van Raamsdonk.

It is proposed here to introduce two new subgenera. This would lead to four subgenera: subgenus Tulipa with seven sections, subgenus Eriostemones Raamsd. with three sections, and subgenus Clusianae (Baker) Zonn. and subgenus
Orithyia (D. Don) Baker with one section each. These four subgenera are based on strong differences in DNA $2 C$ values, morphological differences (Van Raamsdonk 1992; Van Raamsdonk and De Vries 1995), molecular studies at Kew (Wilford 2006), and absence of crossability relations (van Eyk et al. 1991). The eight series of section Eichleres (A.D. Hall) Raamsd. showed large differences in geographic distribution (Hoog 1973) and morphology (Van Raamsdonk and De Vries 1995). Therefore they are placed here in four separate sections, Lanatae (Raamsd.) Zonn. with eight species from mainly the Pamir-Alay, 
Table 2 All species of Tulipa with their nuclear DNA content and ploidy, compared with the systematics of Van Raamsdonk et al. (1997)

Subgenera, sections, and species $\quad 2 C$ DNA (pg) SD Ploidy ${ }^{\mathrm{a}} \quad$ Subgenera, sections, and species of Tulipa

of Tulipa in this article acc. to van Raamsdonk et al. (1997)

\section{Genus Tulipa L.}

Subgenus Clusianae (Baker) Zonn.

\section{Section Clusianae Baker}

T. clusiana de Candolle

T. linifolia Regel

T. montana Lindley

Subgenus Orithyia (D. Don) Baker

Section Orithyia (D.Don) Vved.

T. heterophylla (Regel) Baker

T. uniflora (L.) Besser ex Baker

T. heteropetala Ledeb.

\section{Subgenus Tulipa}

Section Kolpakowskianae Raamsd. ex Zonn.\& Veldk.

T. lemmersii Zonn., A. Peterse, J. de Groot sp. nov.

T. nitida $\mathrm{Hoog}$

T. zenaidae Vved.

T. tetraphylla Regel

T. iliensis Regel

T. brachystemon Regel

T. kolpakowskiana Baker

T. korolkowii Regel

T. borszczowii Baker

T. lehmanniana Mercklin

T. hissarica Popov \& Vved.

T. anisophylla Vved.

T. korshinskyi Vved.

T. ferganica Vved.

T. altaica Pall ex Spreng.

T. ostrowskiana Regel

\section{Section Multiflorae (Raamsd.) Zonn.}

T. subpraestans Vved.

T. heweri Raamsd.

T. praestans Hoog

Section Lanatae (Raamsd.) Zonn. comb.et stat. nov.

T. hoogiana B. Fedtsch.

T. sp.

T. fosteriana Hoog

T. lanata Hoog

T. affinis Botschantz.

T. tubergeniana Hoog

T. tubergeniana Hoog

T. carinata Vved.

Section Vinistriatae (Raamsd.) Zonn.

T. micheliana Hoog

T. vvedenskyi Botschantz.

T. mogoltavica Popov \& Vved.

T. alberti Regel

T. butkovii Botschantz.
37.5

38.3

39.1

36.4

38.6

39.8

40.1

41.0

41.0

41.8

41.5

41.6

42.3

42.8

43.3

44.0

44.8

44.9

77.7

45.7

47.7

50.6

48.8

50.8

51.9

52.5

52.5

53.5

54.2

48.0

51.4

51.6

52.4

53.9

\section{Genus Tulipa}

\section{Section Clusianae}

$1.92 X / 4 X / 5 X \quad$ T. clusiana de Candolle

$\begin{array}{lll}1.7 & 2 X & T \text {. linifolia Regel }\end{array}$

$1.52 X \quad$ T. montana Lindley

$\begin{array}{lll}0.3 & 2 X & \text { nd } \\ 0.4 & 2 X & \text { nd } \\ 0.6 & 2 X & \text { nd }\end{array}$

Subgenus Tulipa

Section Kolpakowskianae

$0.52 X \quad$ nd

$0.82 X \quad$ T. tetraphylla ssp. ostrowskiana (Regel) Raamsd.

$1.02 X \quad$ T. lehmanniana Mercklin

$1.22 X / 4 X \quad$ T. tetraphylla Regel

$0.92 X \quad$ T. altaica Pall ex Spreng.

$0.92 X \quad$ nd

$1.72 X / 4 X \quad$ T. altaica Pall ex Spreng.

$0.92 X \quad$ T. tetraphylla Regel

$1.42 X \quad$ nd

$1.02 X / 3 X \quad$ T. lehmanniana Mercklin

$1.32 X \quad$ T. hissarica Popov \& Vved.

$1.22 X \quad$ T. altaica Pall ex Spreng.

$0.72 X \quad$ T. altaica Pall ex Spreng.

$\begin{array}{lll}1.8 & 2 X & \text { T. altaica } \text { Pall ex Spreng. }\end{array}$

$1.62 X / 4 X \quad$ T. altaica Pall ex Spreng.

$1.94 X \quad$ T. tetraphylla ssp. ostrowskiana (Regel) Raamsd.

\section{Section Multiflorae/Glabrae}

$\begin{array}{lll}1.2 & 2 X & \text { T. subpraestans Vved. } \\ 1.2 & 2 X & \text { T. heweri } \text { Raamsd. } \\ 1.0 & 2 X & \text { T. praestans } \text { Hoog }\end{array}$

Series Eichleres/Lanatae

$1.22 X / 3 X \quad$ T. tubergiana Hoog

$0.92 X \quad$ T. eichleri horT.

$1.02 X \quad$ T. fosteriana Hoog

$0.42 X / 3 X \quad$ T. lanata Hoog

$1.32 X \quad$ nd

$1.12 X \quad$ T. tubergeniana Hoog

T. ingens Hoog

$1.22 X \quad$ nd

Series Vinistriatae/Spiranthera/Undulatae

$0.82 X \quad$ T. eichleri var. micheliana (Hoog) Raamsd.

$\begin{array}{lll}1.7 & 2 X & \text { T. albertii Regel }\end{array}$

$1.52 X \quad$ T. greigii Regel

$1.62 X \quad$ T. albertii Regel

$\begin{array}{lll}1.8 & 2 X & \text { T. albertii Regel }\end{array}$ 
Table 2 continued

Subgenera, sections, and species $\quad 2 C$ DNA (pg) SD Ploidy ${ }^{\mathrm{a}} \quad$ Subgenera, sections, and species of Tulipa

of Tulipa in this article

T. greigii Regel

Section Spiranthera Vved. ex Zonn. \& Veldk.

T. berkariensis Ruksans nom ill.

T. kaufmanniana Regel

T. tschimganica Botschantz.

T. dubia Vved.

\section{Section Tulipanum Reboul}

T. undulatifolia Boiss.

T. armena Boiss.

T stapfii Turill

T. kuschkensis B.Fedtsch.

T. systola Stapf

T. schmidtii Fomin

T. julia K. Koch

T. cypria Stapf ex Turill

T. praecox Tenore

T. praecox Tenore

T. aleppensis Boiss.

\section{Section Tulipa}

T. schrenkii Regel

T. suaveolens Roth

T. eichleri Regel

T. sosnovskyi Akhv.\& Mirz.

T. florenskyi Woronow

T. karabachensis Grossh.

T. hungarica Borbas

T. rhodopaea (Velen.) Velen.

T. gesneriana L.

T. gesneriana L.

\section{Subgenus Eriostemones (Boiss.) Raamsd.}

\section{Section Sylvestres Baker}

T. patens C.Agardh ex Schult\&Schult. f

T. biebersteiniana Schult. $\mathrm{f}$

T. hageri Heldreich

T. orphanidea Boiss. ex Heldr.

T. orphanidea ssp. doerfleri (Gand.) Zonn.

T. orphanidea ssp. whittallii (Dykes) Zonn.

T. sylvestris $\mathrm{L}$.

T. sylvestris ssp. australis (Link) Pamp.

T. primulina Baker

T. sprengeri Baker

T. celsiana DC

Section Biflores A.D.Hall ex Zonn. \& Veldk.

$T$. sp. nov. from Dzjungaric Ala-Tau

T. tarda Stapf

T. dasystemon Regel

T. regelii Elwes.

T. sp. nov. from Altai, Kazakhstan

$\begin{array}{llll}53.4 & 1.0 & 2 X & \text { T. greigii Regel } \\ 50.9 & 0.3 & 2 X & \text { T. kaufmanniana Regel } \\ 53.2 & 1.4 & 2 X / 3 X & \text { T. kaufmanniana Regel } \\ 53.7 & 0.9 & 2 X & \text { T. tschimganica } \text { Botschantz. } \\ 54.7 & 0.9 & 2 X & \text { T. dubia } \text { Vved. }\end{array}$

Section Tulipanum/Aureofasciatae

$51.2 \quad 2 X / 3 X \quad$ nom. nud.

$51.8 \quad 1.7 \quad 2 X / 3 X \quad$ T. armena Boiss.

$52.7 \quad 0.6 \quad 2 X \quad$ T. julia K. Koch

$53.3 \quad 0.4 \quad 2 X \quad$ T. kuschkensis B.Fedtsch.

$56.3 \quad 1.12 X \quad$ T. systola Stapf

$\begin{array}{lll}57.9 & 2 X \quad \text { nd }\end{array}$

$\begin{array}{llll}61.6 & 2.0 & 2 X & \text { T. julia } \text { K. Koch }\end{array}$

$\begin{array}{llll}78.8 & 0.8 & 3 X & \text { nd }\end{array}$

$88.3 \quad 0.6 \quad 2 X / 3 X \quad$ T. praecox Tenore

$1.33 X \quad$ T. aleppensis Boiss.

Section Tulipa/Luteo-apiculatae

$61.5 \quad 1.32 X \quad$ T. suaveolens Roth

$\begin{array}{llll}61.7 & 1.7 & 2 X & \text { T. suaveolens } \text { Roth }\end{array}$

$\begin{array}{lll}62.7 & 2 X & \text { nd }\end{array}$

$63.4 \quad 1.6 \quad 2 X \quad$ T. sosnovskyi Akhv.\& Mirz.

$64.2 \quad 2 X \quad$ nd

$65.6 \quad 1.6 \quad 2 X \quad$ T. suaveolens Roth

$65.6 \quad 2.3 \quad 2 X \quad$ T. hungarica Borbas

$68.62 .12 X \quad$ T. hungarica ssp. rhodopaea (Velen.) Raamsd.

$68.6 \quad 1.22 X \quad$ T. gesneriana L.

$2 X \quad T$. didieri Jord.

Subgenus Eriostemones

Section Australes

55.0

56.7

57.7

59.6

88.7

115.9

120.9

62.0

64.2

64.6

66.0

$1.32 X$

T. biebersteiniana Schult. f

$1.42 X$

T. biebersteiniana Schult. f

nd $2 X / 3 X$

$1.32 X$

T. hageri Heldreich

$1.53 X$

$3.24 X$

$3.23 X / 4 X$

T. orphanidea Boiss. ex Heldr.

T. doerfleri

T. whittallii Dykes

$1.52 X \quad$ T. australis Link

$1.92 X \quad$ T. primulina Baker

$\begin{array}{lll}1.8 & 2 X \quad \text { nd }\end{array}$

$0.42 X \quad$ T. australis Link

Section Biflores

$\begin{array}{llll}48.0 & 0.4 & 2 X & \text { nd }\end{array}$

$\begin{array}{llll}51.5 & 1.3 & 2 X & \text { T. tarda Stapf }\end{array}$

$51.5 \quad 1.32 X \quad$ T. dasystemon Regel

$\begin{array}{llll}52.4 & 1.5 & 2 X & \text { nd }\end{array}$

$\begin{array}{llll}52.4 & 0.4 & 3 X & \text { nd }\end{array}$ 
Table 2 continued

\begin{tabular}{|c|c|c|c|c|}
\hline $\begin{array}{l}\text { Subgenera, sections, and species } \\
\text { of Tulipa in this article }\end{array}$ & $2 C$ DNA (pg) & SD & Ploidy $^{\mathrm{a}}$ & $\begin{array}{l}\text { Subgenera, sections, and species of Tulipa } \\
\text { acc. to van Raamsdonk et al. (1997) }\end{array}$ \\
\hline T. neustruevae Pobed. & 54.8 & 0.7 & $2 X$ & T. neustruevae Pobed. \\
\hline T. biflora Pallas & 55.7 & 1.8 & $2 X / 4 X$ & T. biflora Pallas \\
\hline T. turkestanica Regel & 56.2 & 0.5 & $2 X / 4 X$ & T. turkestanica (Regel) Regel \\
\hline T. polychroma Stapf & 57.0 & 2.0 & $2 X / 3 X$ & T. polychroma Stapf \\
\hline T. sogdiana Bunge & 57.4 & 1.4 & $2 X$ & T. sogdiana Bunge \\
\hline T. dasystemonoides Vved. & 57.7 & 1.3 & $2 X$ & T. dasystemon Regel \\
\hline T. orithyioides Vved. & 58.3 & 1.7 & $2 X$ & T. orithyioides Vved. \\
\hline T. orthopoda Vved. & 59.3 & 1.4 & $2 X$ & nd \\
\hline T. binutans Vved. & 59.4 & 1.5 & $2 X$ & T. sogdiana Bunge \\
\hline T. turcomanica B.Fedtsch. & 76.3 & 1.2 & $3 X$ & nd \\
\hline Section Saxatiles (Baker) Baker & & & & Section Saxatiles \\
\hline T. cretica Boiss. \&Heldr. & 60.5 & 2.2 & $2 X$ & nd \\
\hline T. saxatilis A.D.Hall & 93.1 & 1.7 & $3 X$ & T. saxatilis A.D.Hall \\
\hline T. saxatilis ssp. bakeri (Hall) Zonn. & 62.7 & 1.3 & $2 X$ & T. bakeri A.D.Hall \\
\hline T. pulchella (Fenz ex Regel) Baker & 61.7 & 0.7 & $2 X$ & T. pulchella (Fenz.ex Regel) Baker \\
\hline T. humilis Herb. & 65.0 & 0.7 & $2 X$ & T. humilis Herb. \\
\hline T. humilis Herb. & & & $2 X$ & T. aucheriana Baker \\
\hline \multicolumn{5}{|l|}{ Genus Amana Honda } \\
\hline A. edulis (Miq.) Honda & 114.7 & 4.3 & $4 X$ & nd \\
\hline A. erythronioides (Baker) Tan \& Hong & 50.7 & 3.0 & $2 X$ & nd \\
\hline
\end{tabular}

a Ploidy was based on published chromosome numbers and here determined genome sizes $n d$ Not determined

Vinistriatae (Raamsd.) Zonn. from mainly the Tien Shan with six species, section Spiranthera Vved. ex Zonn. and Veldk. comb. nov. with four species and Multiflorae (Raamsd.) Zonn. comb. nov. with three species (Table 2). Tulipa armena Boiss. from the Caucasus and surroundings is added to section Tulipanum Reboul. Crosses between these four subgenera, to the extent they have been tried, have never been successful (Van Raamsdonk and De Vries 1995).

This scheme with four subgenera and 12 sections has the attraction of simplicity and does not suggest any higher order relationship. The latter can better wait until the species have been sequenced. Details of the new nomenclature for the infrageneric taxa are in development (Veldkamp and Zonneveld, submitted). The genus Amana Honda (syn: Tulipa) is considered to be a separate genus as molecular sequence data have shown that even the mainly American genus Erythronium L. is closer to Tulipa than Amana (Rudall et al. 2000). All species are grouped by increasing DNA $2 C$ value (Table 1 ) into sections, and some species are moved from one section to another. Notes on the sections discussed below follow this classification. A low intraspecific variation is found in most cases. The interspecific variation shows that genome size in diploid Tulipa ranges from 32 to $69 \mathrm{pg}$ (Tables 1, 2).

\section{Genus Tulipa L.}

Subgenus Clusianae (Baker) Zonn., comb. nov. Lectotype: T. clusiana DC. (Veldkamp and Zonneveld, submitted).

Section Clusianae Baker: T. clusiana DC., T. linifolia Regel, T. montana Lindl., T . maximowiczii Regel (not seen).

Subgenus Clusianae is distinguished from the remaining tulips by its low amount of DNA -32 pg instead of 36$69 \mathrm{pg}$ for the other tulips (Table 1). Moreover, meiosis takes place at the start of spring in March in contrast to other species of section Tulipa where it occurs in the period September-November (Darlington, fide Hall 1940). Also DNA studies at Kew (Wilford 2006) have shown that the three species are clearly separated from the rest of Tulipa. All three species have a near identical DNA content, dissimilar to any other species, suggesting that they are closely related. Twelve accessions were grouped under T. clusiana (syn: T. aitchisonii A.D. Hall, T. stellata Hook., T. chrysantha Boiss.), 11 under T. linifolia Regel (syn: T. batalinii Regel and T. montana Hort. yellow form), and 5 accessions under $T$. montana Lindl. (syn: T. wilsoniana Hoog). The true $T$. maximowiczii Regel was not available for study as it could not be maintained in culture 
(W. Lemmers, personal observation). It is morphologically similar to $T$. linifolia but has upright leaves, acuminate tepals and at the base of the tepals, a small blue-black spot flanked by a white to cream edge. There is, moreover, a striking difference in karyotypes as it is the only species with $2 n=22$ (Botschantzeva 1962). The plants in culture under $T$. maximowiczii turned out to be $T$. linifolia. According to Botschantzeva (1962), T. wilsoniana differs both morphologically and in typical features of its karyotype from $T$. linifolia. Tulipa wilsoniana grows in the Kopet Dagh, Kazhakstan, and only red flowered forms are present. The yellow T. batalini is considered by A.D. Hall (1940) to be a form of $T$. montana but according to Van Raamsdonk and De Vries (1995), it is a form of T. linifolia. As both have the same amount of nuclear DNA, it was impossible to discriminate them on that account. In the Elburz Mountains, Iran, both yellow and red forms of T. linifolia occur, often in mixed colonies in roughly equal numbers (Hewer 1974). The opinion of Van Raamsdonk and De Vries (1995) is followed here, and T. batalinii and the yellow form of $T$. montana Hort. are treated as $T$. linifolia. T. linifolia differs from $T$. montana by its more numerous (7-9), more linear leaves, crowded at the base, and they have a low crossibility (Van Raamsdonk and De Vries 1995). Several forms of $T$. linifolia were in culture in the Netherlands but only the one that provided the largest number of new bulbs each year has survived (W. Lemmers, personal communication).

The references to $T$. montana [often described as var. julia (K. Koch) Baker] in Turkey, Lebanon, and Syria should be referred to T. julia K. Koch or T. systola Stapf, both distinguished by having a substantially higher amount of nuclear DNA.

Six of the $T$. clusiana accessions had a cultivar name but were indistinguishable in DNA $2 C$ value from authentic $T$. clusiana. Van Raamsdonk and De Vries (1995) report that crosses between the three species of subgenus Clusianae can be successful. Diploid T. clusiana has been referred to f. cashmeriana (A.D. Hall) Raamsd. and tetraploids to f. diniae Raamsd. All triploids of $T$. clusiana found were cultivars, supposedly from crossing diploids and tetraploids. Subgenus Clusianae can be found from Kashmir, northern Pakistan, and northern Afghanistan to northwestern Iran (Dasgupta and Deb 1985). A pentaploid form can be found naturalized from Turkey to Spain. It is very uniform and could constitute a single clone. Its stoloniferous habit could be an adaptation to cultivated fields, similar to that found in some other species. A $T$. clusiana that was received as $T$. stellata iswith 41 pg-probably an aneuploid as was earlier reported by Hall (1940).

Tulipa subgenus Orithyia (D. Don) Baker. Lectotype: Tulipa uniflora (L.) Besser ex Baker
Section Orithyia (D. Don) Vved.: T. heterophylla (Regel) Baker, T. heteropetala Ledeb., T. uniflora (L.) Besser ex Baker.

Next to T. clusiana with $32 \mathrm{pg}$, the species of this section have the lowest amount of nuclear DNA ranging from 38 to $39 \mathrm{pg}$ (Table 1 ).

Species of the subgenus Orithyia are mainly confined to Siberia, northwestern China, and adjacent Kazakhstan. The stigma of most tulip species "sits" on the ovary with no discernable style. The species of subgenus Orithyia are characterized by a style nearly as long as the ovary and bulb tunics that are usually naked on the inside (Vvedenskii 1935). These three species have small, yellow flowers. Moreover, they have a DNA $2 C$ value of only 38-39 pg and are found at the eastern/northern border of the tulip area, suggesting an isolated position. Tulipa uniflora from northern Kazakhstan and adjacent countries has rounded tepal tops and has a hanging bud. Tulipa heteropetala from the same area has pointed tepals, anthers with a small black tip, and a style protruding clearly above the anthers. The flowers of $T$. heterophylla from southern Kazakhstan are held in a more or less horizontal position when flowering. It is found growing together with $T$. dasystemon (Regel) Regel (Lemmers and de Groot, personal communication). Molecular studies at Kew suggest they are rather deviating from subgenus Tulipa (Wilford 2006). Therefore, they are placed anew in a separate subgenus. Tulipa sinkiangensis Z.M. Mao and T. thianshanica Regel, which likely belong to this subgenus, were not available for study.

\section{Tulipa subgenus Tulipa}

Section Kolpakowkianae, Raamsd. ex Zonn. and Veldk., sect. nov. Type: Tulipa kolpakowskiana Regel. (T. altaica Pall. ex Spreng., T. anisophila Vved., T. brachystemon Regel, T. ferganica Vved., T. hissarica Popov \& Vved., T. iliensis Regel, T. kopalkowskiana Regel, T. korolkowii Regel, T. korshinskyi Vved., T. lehmanniana Merckl., T. lemmersii Zonn., A. Peters \& J. de Groot, T. nitida Hoog, T. ostrowskiana Regel, T. tetraphylla Regel).

After subgenus Clusianae with $32 \mathrm{pg}$ and subgenus Orithyia with $38 \mathrm{pg}$, the species of this section have the lowest amount of nuclear DNA ranging from 36 to $45 \mathrm{pg}$ (Table 1). This fits with the results of Van Raamsdonk and De Vries (1995) who found less than 2\% heterochromatin in section Kopalkowskiana. This contrasts with sections Tulipanum and Tulipa were more than $20 \%$ heterochromatin was found and where accordingly more than $60 \mathrm{pg}$ was measured here. Also Newton (1927) and Blakey and Vosa (1962) found that the chromosome size of T. kopalkowskiana was in between subgenus Clusianae and the other Tulipae. Species of this section can be divided into two groups (J. de Groot, personal communication), those that are multiflowered, with yellow flowers and hairy scapes: T. iliensis, T. ferganica, T. anisophylla, and 
T. tetraphylla (T. tetraphylla glabrous). The others are single-flowered with red or yellow flowers and have glabrous scapes. Exceptions are T. korolkowii, which has only red (pink) flowers and $T$. hissarica which only has yellow flowers. Hall states that $T$. lehmanniana has affinity to T. korolkowii and the latter to T. ostrowskiana, and Van Raamsdonk and De Vries (1995) also place it in synonymy with $T$. ostrowskiana. However having seen them in the wild in Kazakhstan in April 2007, there is no doubt that they are different species. The pink form of T. korolkowii, described as $T$. rosea Vved., is considered conspecific as f. rosea. This is based on my observation of an occasional red form amidst pink $T$. rosea in the wild. Tulipa kopalkowskiana is a mainly yellow-flowering lowland form; higher up, the tetraploid (amphidiploid?; Botschantzeva 1962) T. ostrowskiana with mixed colors is found; and stiller higher up, the mainly yellow-flowered $T$. tetraphylla (Botschantzeva 1962). The karyotype of T. tetraphylla is unique (Botschantzeva 1962) with some chromosome pairs more "related" to those of subgenus Eriostemones. Moreover, garden tulips have never been crossed successfully with species of section Kopalkowskianae (Van Raamsdonk and De Vries 1995).

In nature, T. kopalkowskiana is said to hybridize frequently with $T$. tetraphylla and $T$. ostrowskiana (Vvedenskii 1935). It is remarked that very few true intermediates are found, in most cases there seems to be introgression of some characters (flower color?) only. It could be that this observation is based on the premise that T. kolpakowskiana has yellow flowers, but it can also have reddish colors, obviating the need for the involvement of hybridization. Only a single triploid was found among 30 accessions of section Kopalkowskianae. Botschantzeva suggests that one of the parents of $T$. ostrowskiana is T. kopalkowskiana. Van Raamsdonk et al. (1995) have found that $T$. altaica, despite a high morphological similarity, does not cross with $T$. kopalkowskiana, considered by them to be a synonym of it. They are kept here as separate species as they clearly differ in DNA $2 C$ values. $T$. iliensis with a hairy scape and the tetraploid $T$. tetraphylla with a glabrous scape both have bell-shaped and typically colored flowers: yellow with a red back upon which there is a green stripe. Based on their similar DNA $2 C$ values, $T$. anisophylla could be united with $T$. altaica, but that is not executed here. Tulipa schrenkii was also placed in this section by Botschantzeva (1962), but its amount of nuclear DNA indicates that it should be placed in section Tulipa as was already done by Van Raamsdonk and De Vries (1995).

From the nearly vertical rocks in the Mashad pass, south of Dzabaghly and east of Chimkent in Kazakhstan, a tulip was found that looked most like T. kolpakowskiana. However, it has a 5 pg lower amount of DNA, 36 instead of $41 \mathrm{pg}$, and flowers 14 days later. As there is no other species with a similar amount of DNA, it is described below as a new species: T. lemmersii Zonn., A. Peterse, J. De Groot.

Tulipa section Lanatae (Raamsd.) Zonn. stat. et comb. nov. Type: Tulipa lanata Regel (including series Lanatae Raamsd. and series Eichleres A.D. Hall, p.p.). ([T. affinis Botschantz., T. carinata Vved., T. sp. (T. eichleri hort.), T. fosteriana Irving, T. hoogiana B. Fedtsch., T. lanata Regel, T. tubergeniana Hoog)].

Van Raamsdonk et al. (1997) placed the largest number of species in section Eichleres (A.D. Hall) Raamsd. and divided this further into eight series of which five are monotypic. These eight series are combined here into four sections. This is based on their DNA $2 C$ values (Table 1) and also on the results of crossability studies of Van Raamsdonk et al. $(1995,1997)$ and on geographical proximity. Here series Multiflorae (Raamsd.) Zonn. (including series Glabrae Raamsd.), series Spiranthera Vved. ex Zonn. and Veldk. and Vinistriatae (Raamsd.) Zonn. (including series Undulatae Raamsd.) are given sectional status. Section Lanatae (Raamsd.) Zonn. is now confined to species from the Pamir-Alay and includes series Lanatae sensu Raamsd. Tulips in culture as T. eichleri, without locality data, clearly belong to the tulips around section Lanatae, including in terms of nuclear DNA content. However authentic material of T. eichleri from Azerbaijan turned out to have $10 \mathrm{pg}$ more DNA, so fits better with the other tulips of the Caucasus in section Tulipa. So the section Eichleres is now without its type plant and is named here after its most widespread species, $T$. lanata. In T. lanata, diploid and triploid forms are found. The latter, confined to cultivated fields and roofs of temples, is rather uniform morphologically. Due to morphological similarities, T. hoogiana has been considered to represent a synonym of T. tubergeniana (Van Raamsdonk and De Vries 1995). Their more distant relationship is suggested on the basis of their DNA $2 C$ values, strongly felted tunics [T. tubergeniana had only a few silky hairs in culture, but apparently is strongly felted in nature (Botschantzeva 1962)] and their different geographical distributions. Van Raamsdonk and De Vries (1995) found T. hoogiana also to be distinct from $T$. fosteriana. Hall (1940) remarked that T. lanata, T. ingens Hoog, T. tubergeniana, and T. fosteriana might constitute one variable species. The similarity in average DNA content for members of this section corroborates their close systematic relationships. Furse (1966) remarks that, whereas $T$. micheliana has in nature only a black blotch on the tepals, in culture this black blotch often has a yellow edge, showing again the variability of this character. Tulipa micheliana is included here in section Vinistriatae.

Tulipa section Multiflorae (Raamsd.) Zonn. comb. nov. Type: Tulipa praestans Hoog (T. heweri Raamsd., 
T. praestans Hoog, T. subpraestans Vved.; the latter from series Glabrae Raamsd.).

Tulipa praestans is nearly completely generatively isolated from the other species in section Eichleres Zonn. and Veldk. This separate and revised section seems justified on morphological grounds (multiflowered, tough bulb skin, absence of a basal tepal spot) but also based on the lower amount of DNA compared with the other species of section Eichleres.

Tulipa section Vinistriatae (Raamsd.) Zonn. comb. nov. (including series Undulatae Raamsd.). Type: Tulipa greigii Regel. (T. affinis Botschantz., T. alberti Regel, T. butkovii Botschantz., T. carinata Vved., T. greigii Regel, T. micheliana Hoog, T. mogoltavica Popov \& Vved., T. vvedenskyi Botschantz.).

Tulipa greigii usually has anthocyan markings on the leaves, a character that occasionally can also be found in the largely sympatric other species of this section in the Tien Shan. Tulipa greigii is itself not that easy to cultivate, being sensitive to the fungus Fusarium. As it is fairly easy to cross with $T$. kaufmanniana, T. alberti, and T. vvedenskyi, it is mainly the hybrids that are in culture. For T. micheliana from the Kopet Dagh, it is reported that plants with striped and unicolored leaves grow in the same locality (M. van den Brink, personal communication). Therefore the above-mentioned species are included here in section Vinistriatae. Botschantzeva (1962) placed, based on morphological grounds, T. alberti, T. micheliana, $T$. greigii, and T. mogoltavica together in the same group. The rare yellow forms of both $T$. greigi and $T$. alberti can have a crimson blotch on the inner side of the petal (also in T. heweri and T. kopalkowskiana), a character supposed to be typical of $T$. tschimganica. Tulipa alberti with occasionally a dark leaf with some green stripes is not synonymous with $T$. vvedenskyi. The latter has long narrow wavy leaves, whereas $T$. alberti has short broad gray leaves close to the ground. Characteristically the three inner tepals of T. alberti are folded to the inside and the three outer tepals are folded to the outside (W. Lemmers, personal communication).

Tulipa section Spiranthera Vved. ex Zonn. and Veldk. Type T. kaufmanniana Regel. (T. berkariensis Ruksans nom. invalid., T. dubia Vved., T. kaufmanniana Regel, T. tschimganica Botschantz.).

Tulips of section Spiranthera were originally named for anthers that open up from apex to bottom, becoming contorted afterwards. They usually have flowers with a yellow or white background, in contrast to section Vinistriatae, whose flowers are all mainly red. Tulipa dubia is very similar to the sympatric $T$. kaufmanniana (Ivaschenko 2005) differing mainly in its smaller overall size and lessspiraling anthers. It could be classified as a subspecies of T. kaufmanniana. Tulipa dubia crosses in the wild with
T. kaufmanniana and the result of such a cross may be T. tschimganica. It fits with its in-between amount of nuclear DNA. Also numerous hybrids between T. kaufmanniana and T. greigii are reported from the Karshan-tau mountains (Vvedenskii 1935, in transl.). The invalid T. berkariensis from the Berkara valley and other places in Kazakhstan has a lower amount of nuclear DNA than authentic T. kaufmanniana from Uzbekistan and might present a new species.

Tulipa section Tulipanum Reboul. Type: Tulipa agenensis DC (as T. oculus-solis St. Amans, nom. invalid.) (T. aleppensis Boiss. ex Regel, T. armena Boiss., T. cypria Stapf ex Turill, T. julia K. Koch, T. praecox Ten., T. stapfii Turill, T. schmidtii Fomin, T. systola Stapf, T. undulatifolia Boiss.).

These species are reported from Turkey, northwestern Iran, northern Iraq, Lebanon, Israel, Egypt, and Syria. They are thus largely geographically isolated from the Asiatic centers of diversity. Tulipa armena is added to this section based on its DNA content, although it does not have a tunic thickly lined with wool as have the other species. Strongly felted bulb tunics are not only found in section Tulipanum but also in section Lanatae, including T. lanata, T. hoogiana, and T. tubergeniana. A thickly felted tunic, although it could be discriminatory in the field, is often strongly influenced by cultural conditions. It does not seem to offer a strong argument to attribute species or to separate sections.

Tulipa undulatifolia, with $51.2 \mathrm{pg}$, seems related to T. armena with 51.8 pg. Tulipa boeotica Boiss. \& Heldr. is reported in Flora Europaea to have $2 n=24$. However a plant from Didyma, Peloponnesus, Greece, turned out to be a triploid with $76.8 \mathrm{pg}$. It differs in that respect both from T. praecox and T. aleppensis and is here suggested to be the triploid form of $T$. undulatifolia.

According to Hall (1940), Tulipa armena is synonymous with T. gesneriana. M. van den Brink (personal communication, 2008) showed me pictures of T. armena from Erzurum, Turkey, with pointed petals like T. schrenkii. Therefore it is understandable that Phillips and Rix (1981) also assumed a close relationship between $T$. armena from Erzurum and T. schrenkii. However, these opinions are not corroborated by DNA content values. Van Raamsdonk and De Vries (1995) placed T. armena in section Tulipa, but noted that it differed from the Neotulipae. Also Booy and Van Raamsdonk (1998) remark that the esterase patterns from $T$. armena are different from those of $T$. gesneriana. The results here indicate, for 10 accessions of $T$. armena (two accessions identical to the ones used by van Raamsdonk), that $T$. armena is not related to $T$. gesneriana or T. schrenkii. Moreover, it fits best with its $51.8 \mathrm{pg}$ in section Tulipanum and not in section Tulipa, where all species have more than $62 \mathrm{pg}$ of DNA. 
Two accessions received as " $T$. armena" were found to have about $62 \mathrm{pg}$. These are likely wrongly diagnosed and are placed here with the sympatric $T$. julia, which also has pointed petals (M. van den Brink, personal communication, 2008), a similar yellow-edged black blotch at the base of the tepals, and the same amount of nuclear DNA. This might explain the suggestion of Hall (1940), Van Raamsdonk and De Vries (1995), and Phillips and Rix (1981) that "T. armena" has a close relationship with section Tulipa. T. galatica Hort. investigated here has about $68 \mathrm{pg}$. Up to 12 B-chromosomes are reported in T. galatica Freyn (Hall 1940). If the length of these is measured from the published karyotype of $T$. galatica (van Raamsdonk and De Vries 1995) and converted to DNA content, they could contribute $10-15 \%$ of total DNA. If subtracted this would give a value of around $60 \mathrm{pg}$ similar to the "deviating" "T. armena." Both "T. armena" and T. galatica Hort. can then be considered to be forms of $T$. julia. An expedition to Azerbaijan in 2009 was able to locate $T$. schmidtii Fomin described in 1909 of which Hall (1940) says that "its chromosomes are twice as long as in other species." However with its nuclear DNA content of $57.9 \mathrm{pg}$, this is not confirmed. T. schmidtii has some characters in common with $T$. hoogiana, such as strongly keeled leaves; a high number of leaves, up to 8 (up to 12 in T. schmidtii); similar red flowers with a yellowedged black blotch (see figures in Hall 1940); and middle to late flowering period. However the nearly 10-pg difference in nuclear DNA content points to a distant relationship. Moreover T. schmidtii has only a small, threatened territory in Azerbaijan, whereas T. hoogiana is described from the Kopet Dagh on the border of Turkmenistan and Iran and even further east.

According to Marais (1984), the triploids T. praecox and $T$. agenensis may well be clonal selections of the same species. This is not corroborated by their DNA $2 C$ values. Based on the DNA $2 C$ values, $T$. praecox (88.3 pg) might be the triploid form of $T$. systola $(56.1 \mathrm{pg})$, and $T$. aleppensis (95.3 pg) might be a triploid $T$. juliae (62.0 pg) [already suggested by Van Raamsdonk and De Vries (1995)]. It cannot be excluded that both are allo-triploids. The two triploid accessions $T$. praecox and $T$. aleppensis, with about 88 and $95 \mathrm{pg}$, cannot be derived from the socalled Neotulipae with about $69 \mathrm{pg}$ as that would give around $103 \mathrm{pg}$ for the triploid. Moreover, it cannot be excluded that triploids as such have a bulb tunic that is more hairy inside than their diploid counterparts.

Tulipa section Tulipa. Type: Tulipa gesneriana L. [T. eichleri Regel, T. florenskyi Woronow, T. gesneriana L., T. hungarica Borbas, T. confusa Gabrielian, T. schrenkii Regel, T. sosnovskyi Achv. \& Mirz. (formerly of series Luteo-apiculatae Raamsd.), T. suaveolens Roth].

The description of $T$. gesneriana L. s.l., was based on cultivated material, and it is generally considered to cover the late-flowering cultivars, including the so-called Neotulipae from western Europe (Levier 1884; Grey-Wilson and Mathews 1980). It is characterized by large bulbs with a papery tunic with a few addressed hairs near the base and apex, by green buds, often a yellow-edged black base to the (red) tepals, and deep purple pollen. It has about $69 \mathrm{pg}$ of DNA, higher then any other tulip species. "Species" such as T. didieri Jord., T. grengiolensis Thommen, T. marjolettii E.P. Perr. \& Song., and other Neotulipae (Hall 1940) are easily hybridized with cultivars of $T$. gesneriana s.l. (Van Raamsdonk et al. 1995) and are considered escapes from cultivation. Tulipa confusa (syn. T. karabachensis) has yellow, red, and pink flowers, usually without a black blotch at the base of the tepals. It is from Azerbaijan and southeastern Armenia (Shikahogh reserve) and is further characterized by short and comparatively broad leaves. Sometimes it is considered to belong to Neotulipae, and Van Raamsdonk considers karabachensis to be a synonym of $T$. suaveolens, but its DNA $2 C$ value of $65.6 \mathrm{pg}$ contradicts both suggestions. Tulipa sosnovskyi is characterized by prostrate flower buds lying on the leaves or the ground, erecting only at the time of flowering. This plant from Armenia was placed in section Eichleres (series $\mathrm{Lu}$ teo-apiculatae Raamsd.) by Van Raamsdonk and De Vries (1995). Based on its DNA $2 C$ value of $63.2 \mathrm{pg}$, it is better placed in section Tulipa. T. eichleri Regel is considered by Marais (1984) to be a synonym of T. undulatifolia Boiss., both from cultivated fields. In that case, T. undulatifolia must have priority. However, Van Raamsdonk and De Vries (1995) consider T. undulatifolia to be a nomen nudum and accept T. eichleri. T. undulatifolia is described from western Turkey, whereas T. eichleri is described from the Caucasus and western Iran, a 3,000 km distance. Therefore, and because of the more than $10 \mathrm{pg}$ difference in nuclear DNA content, they are placed in different sections. A wild counterpart of $T$. gesneriana has so far not been found (see however, B. J. M. Zonneveld, personal communication).

The early-flowering $T$. suaveolens has bulbs similar to those of $T$. gesneriana, but has hairy stems, buds that often show color, fragrant flowers with a yellow base to the tepals, and yellow pollen. It has on average $61.6 \mathrm{pg}$ of DNA. I propose here that $T$. suaveolens Roth, originally described from the red and yellow cultivar $T$. 'Duc van Tol' and indistinguishable from it, be applied to the cultivated 'Duc van Tol' tulips only. The multicolored T. schrenkii Regel (mainly yellow in northern Kazakhstan, but also white, pink, orange, and pinkish red) has a bell-shaped flower, with a mainly yellow base to the tepals, and mainly yellow anthers. However occasionally plants with a black base and black anthers can also be found (W. Lemmers/J. de Groot, personal communication). It grows in a large area, stretching from the Crimea up to N. Kazakhstan and has the same amount of nuclear DNA as T. suaveolens and is 
likely (one of) its wild progenitor. T. schrenkii from Kazakhstan is reported to be highly fertile with garden tulips (Kudryavtseva in Ivaschenko 2006).

Tulipa subgenus Eriostemones (Boiss.) Raamsd. Lectotype: Tulipa sylvestris $\mathrm{L}$.

Subgenus Eriostemones is characterized by filaments with a bundle of hairs at the basis or with at least a swollen basis. The flowers are funnel-shaped with a slight constriction just above the base. They have mainly lanceolate leaves and three short and three long anthers. Most are multiflowered caused by a branching of the apical bud, a derived character phylogenetically supported by the high amount of DNA found for the species of this section. The occasionally multiple flowers in subgenus Tulipa, on the contrary, grow from the axils of the leaves (Botschantzeva 1962).

Tulipa section Sylvestres (Baker) Baker. Type: Tulipa sylvestris L. (T. biebersteiniana Schult. and Schult. f., T. celsiana DC in R. hageri Heldreich, T. orphanidea Boiss. ex Heldr., T. patens C. Agardh ex Schult. and Schult. f., T. primulina Baker, T. sprengeri Baker, and T. sylvestris L.).

Tulipa biebersteiniana with yellow flowers is often equated with T. sylvestris ssp. australis (Link) Pamp. as they can hardly be distinguished morphologically (Vvedenskii 1935; Hall 1940). However, the difference in DNA content between $T$. biebersteiniana with $56.7 \mathrm{pg}$ and T. sylvestris with 62.3 pg supports the conclusion that these taxa are distinct and worthy of recognition. $T$. patens with a similar geographical spread and DNA $2 C$ value seems closely related to $T$. biebersteiniana. Tulipa patens grows from the Wolga area, via western Siberia and Kazakhstan to the Altai Mountains. According to Mathew (2005), it is morphologically close to T. biflora, but Van Raamsdonk (1992) consider it to be a form of $T$. biebersteiniana. It has white but also often pink flowers and is here considered a good species of the section Sylvestres. Tulipa riparia Knyasev, not measured here, is suggested to be an allotriploid form of $T$. biebersteiniana. Tulipa sylvestris is found in both diploid and tetraploid forms. The diploids are named ssp. australis and the tetraploids ssp. sylvestris. Surprisingly, three accessions from three separate locations in the south of Norway, likely arriving there due to human intervention, turned out to be triploid. They might be derived from a single clone. Tulipa primulina is set apart from $T$. sylvestris ssp. australis. Tulipa primulina has a different geographical distribution in the north of Africa (Algeria) and is also $3 \mathrm{pg}$ higher in DNA $2 C$ value. Moreover, it has the peculiar habit of opening its flowers on a sunny day at the end of the day instead of around noon as all other tulips do. T. hageri is here considered a good species with $57.7 \mathrm{pg}$, a bit lower in DNA $2 \mathrm{C}$ value than T. orphanidea with $59.6 \mathrm{pg}$. Based on cytological evidence and because it has at most $30 \%$ good pollen (fide Hall 1940), T. orphanidea could be an old hybrid of
T. hageri $\times T$. sylvestris as suggested by Hall. This fits with its in-between genome size. Tulipa doerfleri from Crete was earlier placed in section Saxatilis based on geographical arguments. However, based on DNA content and morphology, it is considered here as a triploid ssp. of T. orphanidea. Tulipa whittallii (Dykes) A.D. Hall is here considered a tetraploid subspecies of $T$. orphanidea. $T$. sprengeri has a similar amount of nuclear DNA as T. sylvestris and does not seem strongly related to the other red-flowering plants included in T. orphanidea. Tulipa sprengeri, found in northern Turkey and thought to be extinct in the wild, has maintained itself only in private gardens. There it is grown from seed, but it cannot be grown sustainably from bulbs. Instead of a bundle of hairs on the filaments, these are only swollen at the base in this species.

Tulipa section Biflores A.D. Hall ex Zonn. and Veldk., sect. nov. Type: Tulipa biflora Pall. [T. biflora Pall., T. binutans Vved., T. dasystemon (Regel) Regel, T. dasystemonoides Vved., T. orithyoides Vved., T.orthopoda Vved., T. polychroma Stapf, T. regelii Krassn., T. sogdiana Bunge, T. tarda Stapf, T. turcomanica B. Fedtsch., T. turkestanica (Regel) Regel].

Species of this section look very similar. All have whitish-yellow flowers, mainly differing in the number and consequently size of the flowers on a stalk. T. regelii was placed in a separate section Lophophyllon by Vvedenskii (1935), based on its unique ridges on the leaf. However based on DNA content and flower morphology, it belongs to this section.

Tulipa tarda [syn: T. urumiensis Stapf, contrary to the opinion of Hall (1940) who considered T. urumiensis to be a form of $T$. australis Link] has, with $51.5 \mathrm{pg}$, the lowest amount of DNA in this section. Tulipa neustruevae Pobed is mainly distinguished from $T$. dasystemon by its glossy green leaves, a firmer bulb tunic, and brighter yellow flowers. They do differ slightly in DNA $2 C$ value with 52.0 and $54.8 \mathrm{pg}$ for $T$. dasystemon and $T$. neustruevae, respectively, so here they are kept separate. Apart from a greater amount of nuclear DNA, T. dasystemoniodes is mainly differentiated from $T$. dasystemon by its densely woolly inner surface of the bulb tunic (Hall 1940). It emerges from the soil in a flowering state. All accessions of T. biflora s.l. have a rather narrow range of variation in the nuclear DNA values and similar types of flower, mainly differing in the number and size of the flowers. These data strengthen the viewpoint, based also on morphological evidence, that T. bifloriformis Vved., T. busheana Boiss., and T. talievii Klokov \& Zoz. cannot be upheld as species. Four accessions of "T. biflora" are geographically isolated in the Altai Mountains, Kazakstan. As also their DNA $2 C$ value is $2 \mathrm{pg}$ lower than authentic T. biflora, they might constitute a new species. 
Tulipa sogdiana's main points of difference are its geographic isolation in the desert and the absence of hairs on the filaments, a character also occasionally found in some plants of $T$. biflora. This is not unique for this section as also T. sprengeri and T. orphanidea (Marais 1984) of section Sylvestres are devoid of hairs on the filaments. Tulipa sogdiana with a slightly higher amount of DNA is kept apart here. A triploid form of T. polychroma was found for the first time. Earlier Kroon and Jongerius (1986) reported on a tetraploid and even a hexaploid form of $T$. polychroma. This is so far the only hexaploid reported for the genus. Also the form of T. turcomanica measured here turns out to be triploid.

Tulipa section Saxatiles (Baker) Baker. Type: Tulipa saxatilis Sieber ex Spreng. [T. cretica Boiss. \& Heldr., T. humilis Herb., T. pulchella (Fenzl ex Regel) Baker, T. saxatilis Sieber ex Spreng.].

Section Saxatiles contains four species, T. humilis with 65.0 pg (syn: T. aucheriana Baker, T. violacea Boiss. \& Bushe), T. pulchella (syn: T. kurdica Wendelbo) with $61.7 \mathrm{pg}$, T. saxatilis ssp. bakeri A.D. Hall with $62.5 \mathrm{pg}$, and its triploid form ssp. saxatilis and $T$. cretica with $60.5 \mathrm{pg}$. The last three are confined to Crete. T. bakeri might well be a stabilized hybrid between T. cretica and the diploid form of $T$. saxatilis, but there are no arguments based on DNA $2 C$ value. All three are closely related and have shiny green leaves.

Main flower colors in T. humilis/pulchella are very variable (fide Hall 1940; W. Lemmers, personal communication). In addition, two flower color types are found, those with violet/black (T. pulchella) and those with yellow bases ( $T$. humilis) to the tepals. If the plants from this complex are arranged according to ascending genome size, most of those with a black base to the tepals are in the lower part and those with a yellow base in the higher segment (Table 1). If averaged, they show a strong difference in DNA $2 C$ value with $61.7 \mathrm{pg}$ for $T$. pulchella and 65.0 for $T$. humilis s. str. Therefore they are here considered separate species, and in this we follow Van Raamsdonk et al. (1997).

\section{Genus Amana Honda}

Amana Honda, Bull Biogeogr Soc Japan 6:20, 1935. Tulipa sect. Amana (Honda) Z.M. Mao, Fl Reipubl Pop Sin 14:89 (1980). Type: Amana edulis (Miq.) Honda [Syn: Orithyia edulis Miq., Tulipa edulis (Miq.) Baker]. [A. edulis (Miq.) Honda, A. erythronioides (Baker) Tan, A. anhuiensis (X.S. Shen) D.Y. Tan and D.Y. Hong, A. kuocangshanica D.Y. Tan and D.Y. Hong (the latter two not available for study).

Earlier this genus was considered as belonging to Tulipa but recent DNA investigations have shown that Amana is a distinct genus (Rudall et al. 2000). Two of the four species were measured. They are most likely a diploid and a tetraploid taxon, but the DNA $2 C$ values indicate that the tetraploid taxon is not derived from this diploid.

In Table 2, all taxa have been arranged according to their amount of DNA. They are compared to the most recent treatment of Tulipa of Van Raamsdonk et al. (1992, 1995, 1997). In accordance with earlier results from the genus Helleborus and the genus Narcissus (Zonneveld 2001, 2008), in Tulipa DNA amounts do largely coincide with this earlier infrageneric classification.

\section{Polyploidy}

Ploidy seems to play a bigger role than envisioned in the speciation of Tulipa. Earlier cytological investigation of Tulipa have shown that most species are diploid $(x=12)$ (Kroon and Van Eijk 1977), and this is confirmed here indirectly (Table 1). In addition to the diploid species, triploidy in T. aleppensis, T. clusiana, T. cypria, T. hoogiana, T. kopalkowskiana, T. lanata, T. orphanidea, T. orithyoides, T. praecox, and T. saxatilis was confirmed. For the first time, triploids were also found in T. kaufmanniana, T. polychroma, T. boeotica, T. sylvestris, T. turcomanica, and T. undulatifolia. Moreover, tetraploidy is found in T. biflora, T. dasystemon, T. clusiana, T. orphanidea, T. sylvestris, T. ostrowskiana, T. tetraphylla, and T. turkestanica. Only in T. clusiana were pentaploids found, and in $T$. polychroma hexaploids are recorded (Kroon and Jongerius 1986).

Botschantzeva (1962) stated that the polyploids are concentrated in the middle and upper mountains, whereas the diploids are mainly found in the deserts and lower mountains. The exception must be the stoloniferous plants that are clearly well adapted to cultural conditions as found, e.g., in vineyards. The largest numbers of polyploids are found along the western and eastern boundaries of the distribution area of the genus.

In most cases in tulips, polyploidy is not an argument (any longer) to give a taxon a specific status as also stated by Woods and Bamford (1937). The autotetraploid (Botschantzeva 1962) T. tetraphylla has 79.9 pg. Also diploid forms of $T$. tetraphylla were encountered here. Botschantzeva showed that $T$. ostrowskiana is an amphidiploid, which in the wild easily crosses (back) with T. kopalkowskiana, one of its parents. Only one triploid, possibly resulting from such a cross, was encountered here. The other parent of $T$. ostrowskiana is unknown; however judging from its amount of DNA it must have 77.7$41.3=36.4 \mathrm{pg}$. Plants from the Mashad Pass received as aff. T. kopalkowskiana have a unique amount of DNA with $36 \mathrm{pg}$ and are described below as a new species. They could be the unknown other parent. This would be in 
accordance with the amount of DNA found for T. ostrowskiana. So the suggestion is that $T$. ostrowskiana could be the doubled offspring of $T$. kopalkowskiana with $41 \mathrm{pg} \times$ T. lemmersii Zonn., A. Peterse, \& J. de Groot from Mashad Pass with $36 \mathrm{pg}$. This would give $78 \mathrm{pg}$ for T. ostrowskiana.

The speed and cost effectiveness of measuring nuclear DNA content and its predicative accuracy makes it useful as a tool for identifying Tulipa species of wild origin. This has particular potential for the monitoring of trade in $\mathrm{Tu}$ lipa species, as plants are usually imported as bulbs and only limited identification can be undertaken at this stage of the yearly life cycle. Before identification of a Tulipa species can be undertaken, any bulb has to be grown at a nursery for naming at a later date-a costly and timeconsuming exercise. The nuclear DNA content of samples can be measured in a short time from bulb tissue. Although the DNA content is not unique to every taxon, many species (and some subspecies and varieties) can be identified using this method. Moreover, controversial imports, such as those in which trade is banned, can be distinguished from those for which large-scale commercial trade is allowed.

\section{Evolutionary considerations}

Botschantzeva (1962), considering mainly the tulips of the former Soviet Union and leaving out completely subgenus Clusianae, recognized four main centers of speciation for the genus. The first is the Tien Shan with mainly yellow or at most orange tulips. The second area is the Pamir-Alay, floristically isolated from the Tien Shan with fiercely red tulips. The third center includes the desert and steppes of Kazakhstan extending to eastern Europe. Mountainous Turkmenistan, Iran, and the Caucasus form the fourth center and are floristically connected with the Pamir-Alay. In western Siberia and western China mainly species of subgenus Orithyia are found.

Going from east to west, from northern Pakistan to the Balkans, there seems to be a gradual increase in nuclear DNA content (DNA $2 \mathrm{C}$ value) from $32 \mathrm{pg}$ in section Clusianae to up to $69 \mathrm{pg}$ in section Tulipa. There is a concomitant decrease in the time it takes for meiosis to take place, starting from a dormant bulb. Darlington (in Hall 1940) investigated this in about 20 species. Accordingly, these could be divided in three groups. In subgenus Tulipa, meiosis took place in autumn. (Within this subgenus, the $T$. gesneriana varieties with up to $69 \mathrm{pg}$ underwent meiosis in September/early October, $T$. fosteriana with $52 \mathrm{pg}$ in early November, and the Kolpakowskianae with about $40 \mathrm{pg}$ at the end of November). Then in subgenus Eriostemones meiosis happens in the winter period, December to February. The latest meiosis took place was in subgenus Clusianae with about $32 \mathrm{pg}$ at the start of spring in March (Darlington in Hall 1940).

The relationship shown above between an increase in the amount of nuclear DNA and a decrease in time to meiosis seems in disagreement with nucleotypic theory (Bennett 1972), which would predict that the more DNA the longer it takes to perform mitosis (also meiosis?). Moreover, early or late meiosis does not lead to a large difference in flowering time. Clusianae have rather small bulbs, but they have their leaves at an early time in spring. This might provide the necessary energy input to perform meiosis. However the Gesnerianae could already, early in autumn, rely on the starch storage in their much bigger bulbs. So they might have a sufficient energy level to have an early meiosis. This may have been (inadvertently) encouraged by several centuries of selection for bigger, fast-growing bulbs. It cannot be excluded that the change to a more maritime climate could also have attributed to a different timing of meiosis.

Clusianae are placed in a subgenus separate from the remaining tulips because of their low amount of DNA, $32 \mathrm{pg}$ instead of 36-69 pg for the other tulips. This might indicate a primitive position within the genus. Tulipa clusiana, T. linifolia, and T. montana have identical amounts of DNA, so each one is, on this account, equally eligible for being the most plesiomorphic species. However, the higher variability both in ploidy and flower colors and larger geographical spreading of $T$. clusiana could point to a more ancient origin. Although it has sometimes been suggested in popular literature, tulips of the section Clusianae do not cross with species outside this section. A further distinctive feature is a pair of long chromosomes in which the long arm is more than thrice as long as the short arm, giving a subterminal kinetochore instead of the submedian kinetochore in other tulips (Woods and Bamford 1937). Based on these facts, Clusianae is regarded as a separate subgenus. Subgenus Orithyia is discriminated by a style that is as long as the ovary and a low amount of DNA of about $38 \mathrm{pg}$. They are small plants with yellow flowers and found mainly in the eastern part of the area, the mountains on the border of Kazakhstan and China and adjacent Siberia.

\section{Sections}

The main center of distribution for $T$. clusiana is in Kashmir, Pakistan, northern Iran, Iraq, and northern Afghanistan. The diploid form is from Chitral (northern Pakistan) and Kashmir and grows as far east as the Kumaon Hills in Uttar Pradesh at altitudes of 3,000$4,000 \mathrm{~m}$ but also in northern Afganistan. Probably the plants of T. clusiana found in southern Europe as far south as Spain are escapees from cultivation. They produce 
stolons and might be the pentaploid form. Diploid, tetraploids, and pentaploid forms of $T$. clusiana were measured here, and in culture, triploid forms have been created. For T. linifolia and T. montana thus far only diploid forms are found. Tulipa linifolia has more but narrower leaves compared with T. montana.Tulipa linifolia occupies a more westerly position relative to $T$. clusiana, growing in the Pamir-Alay, Afghanistan, and northern Iran. The related T. montana (including $T$. wilsoniana) can be considered as the most westerly extension, being mainly found in the Kopet Dagh and the Elburz mountains of northwesten Iran. In both cases an increase in DNA was not found.

If an increase in DNA $2 C$ value during evolution is the character to go for, section Kopalkowskiana from the TienShan seems to form the next step with $40-45 \mathrm{pg}$ for the diploids and a concomitant increase in leaf width. The subgenus Orithyia with 38 pg seems to be an early splitoff. From section Kopalkowskiana, a further increase in leaf width seems to have given rise to subgenus Tulipa. This was accompanied by a further increase in DNA $2 C$ value to 50-69 pg and a more westerly position. On the other hand in subgenus Eriostemones with 52-64 pg, an increase in DNA content seems to coincide with an increase in the number of flowers and more northerly locations. In this subgenus $T$. tarda with $51.5 \mathrm{pg}$ might occupy a plesiomorphic position. Both subgenus Eriostemones and section Kopalkowskiana have narrow leaves with often multiple slender stems, seemingly springing from ground level as elongated pedicels, and often drooping buds in common. Sections Lanatae, Multiflorae, Spiranthera, and Vinistriatae with 45-54 pg might be the next step in the evolution. From these, section Tulipanum and section Tulipa of subgenus Tulipa with 52-69 pg might have originated.

\section{Conclusions}

Flow cytometry can be considered as a quick and useful method to produce a systematic data source. Moreover, imported Tulipa bulbs can be used for investigation, precluding the need to grow them to maturity for identification purposes. The difference between the highest and lowest DNA contents in Tulipa is about $37 \mathrm{pg}$. This doubling in DNA content without changing the number of chromosomes must be the result of a vast number of genomic changes. Depending on the size of the total genome, 1 pg amounts to several thousand genes. Therefore, flow cytometry is not a one-character-based taxonomy as the largest genome contains roughly $3.4 \times 10^{10}$ more base pairs than the smallest and has chromosomes that are more than twice as large. Moreover these large differences in the amount of nuclear DNA predict that the hybrids are usually sterile.
The genus Tulipa is here divided into four subgenera: Clusianae, Tulipa, Eriostemones, and Orithyia. The eight series of section Eichleres sensu Raamsd. were united in four sections: section Lanatae, section Multiflorae (including series Glabrae), section Spiranthera, and section Vinistriatae (including series Undulatae). Some species were placed in another section based on morphology but also on their genome size. These are T. armena from section Tulipa to section Tulipanum; T. alberti, T. vvedenskyi, and T. micheliana to section Vinistriatae, also based on the presence of dark stripes on the leaf; T. sosnovskyi and $T$. eichleri from section Lanatae to section Tulipa.

Van Raamsdonk reduced the number of species to 55 (excluding the not investigated subgenus Orithyia). I was in the fortunate position to accompany a tour in April 2007 to visit Kazakhstan, Uzbekistan, and Tajikistan to observe wild tulips. Nearly 30 species were seen, many of them in flower. This company had several people that had repeatedly visited these and other nearby countries. Moreover expeditions to northern Iran and Azerbaijan in 2009 resulted in some new material. Based on intensive discussions and the nuclear DNA content (DNA $2 C$ value) as measured here, several more species are recognized. They are $T$. anisophylla, T. boeotica (undulatifolia), T. brachystemon, T. butkovii, T. celsiana, T. confusa, T. cypria, T. dasystemonoides, T. florenskyi, T. orphanidea ssp. doerfleri, T. ferganica, T. hoogiana, T. iliensis, T. karabachensis, T. kolpakowskiana, T. korolkowii, T. korshinskyi, T. lemmersii sp. nov., T. micheliana, T. mogoltavica, T. nitida, T. orithyoides, T. ostrowskiana, T. patens, T. schmidtii, T. schrenkii, T. stapfii, T. turkestanica, T. undulatifolia, T. vvedenskyi, T. zenaidae.

In synonymy went (compared to van Raamsdonk) T. didieri (included in T. gesneriana), T. doerfleri and T. whitallii (both to $T$. orphanidea) and $T$. aucheriana (to T. humilis).

Plants not investigated by van Raamsdonk et al. (1997) are $T$. affinis, $T$. alberti (the authentic one), T. boeotica, T. borsczowii, T. brachystemon, T. cypria, T. confusa, T. cretica, T. doerfleri, T. eichleri (the authentic one), T. florenskyi, T. goulimyi, T. korolkowii, T. kurdica, T. lehmanniana (the authentic one), T. lemmersii, T. mariannae, T. patens, T. regelii, T. rosea, T. schmidtii, T. sintenisii, T. sprengeri, T. teophrasti, T. zenaidae (the authentic one), and the species of subgenus Orithyia: T. heteropetala, T. uniflora, T. heterophylla, and the genus Amana.

Flow cytometry is shown here to be a very useful tool to indicate the relationship or taxonomic status of Tulipa taxa. Apart from the presence or absence of B-chromosomes, each species has a certain amount of nuclear DNA, which is of systematic value. Taxa clearly different in nuclear 
DNA amounts are considered "good" species. This does not mean that taxa with identical DNA amounts must always be considered as constituting a single species. The nuclear DNA amounts should always be evaluated in combination with morphological data, just as any other taxonomic characters. In general, there is strong congruency between these characters and DNA $2 C$ value. The data presented here for DNA content agree in most respects with the most recent classification of Tulipa (Van Raamsdonk et al. 1997). In some cases, the measured DNA value gives rise to questions about the perceived taxonomic relationship of certain taxa, which are identified as requiring further investigation. Flow cytometry as a taxonomic and diagnostic tool is applicable even in the case of dormant bulbs or sterile plants, and therefore has applications for conservation monitoring.

\section{Lectotypification of the genus Tulipa}

There are differences of opinion on the lectotypification of the genus Tulipa. To summarize the relevant data: Linnaeus (1753) described three species of tulips: T. sylvestris (p. 305), T. gesneriana (p. 306), and T. breyniana (the latter now in the genus Baeometra). It can be argued that $T$. gesneriana fits best his concept of the genus as only T. gesneriana was available in the Clifford Herbarium and probably the only species known to him when he wrote the first edition (1737). Boissier (1882) divided the tulips into two groups although he did not indicate the level: Leiostemones with 12 species, one of which is $T$. gesneriana, and Eriostemones with 13 species, one of which is T. sylvestris. According to the rules, Marais (1984) replaced Leiostemones with Tulipa. Britton and Brown (1913) just stated under Tulipa: typeplant $T$. sylvestris. They seemed just to have chosen the first binomial species in order. Hitchcock and Green (1929) selected T. gesneriana as the lectotype as "the best known of the three species." According to rule 10.5 (Code 2006) the choice of a lectotype may be superseded by a later one if it was based on a largely mechanical method of selection. As only the name is mentioned by Britton and Brown and just the first one of Linnaeus was chosen, this rule seem applicable here. The choice for $T$. gesneriana has been made already by Dasgupta and Deb (1985) and was followed by Jarvis et al. (1992, 1993). Moreover, it follows the general usage of T. gesneriana as the type of the genus Tulipa (Vvedenskii 1935; Hall 1940; Botschantzeva 1962; Marais 1984; Stork 1984; Van Raamsdonk and De Vries 1995; Wilford 2006). T. gesneriana is therefore accepted here as the lectotype as designated by Hitchcock and Green (1929) (see also Veldkamp and Zonneveld, submitted).

\section{Possible new species}

Based on the amount of nuclear DNA, several possible, as yet not described, species emerged. Unfortunately there is not sufficient material yet to describe them in detail and to deposit a type. They include the following:

1. T. $s p$. from the Berkara valley in the Kara-Tau mountains of Kazakhstan. Morphologically it is a smaller form of $T$. kaufmanniana. However, it is geographically isolated from T. kaufmanniana from Uzbekistan and has 2 pg less nuclear DNA. It also has smaller, round bulbs, emerges later, has only two leaves instead of three or four and makes stolons. It was found and described as "a dwarf relative of T. kaufmanniana" in a book by J. Ruksans as T. berkariense (nom. invalid., anglice). Based on these data it could be a new species.

2. T. sp. from Kyrgyzstan. It is a dwarf plant and looks like a plain yellow T. tarda. However, it has more leaves that are purplish coloured and a glabrous scape. Based on these characters and its DNA $2 C$ value it could be a new species or a subspecies of $T$. tarda.

3. T. sp. from Altai, Kazakhstan. It is considered in the local floras (Botschantzeva 1962; Ivaschenko 2005; Pratov et al. 2006) to be T. biflora. However they are geographically isolated from the more westerly growing "true" T. biflora. Moreover they have $2 \mathrm{pg}$ less DNA, so might constitute a new species.

4. T. sp. from Dzjungaric Ala-Tau, Kazakhstan. Based on a plant in seed, it was first considered to be T. busheana, but with only $48 \mathrm{pg}$ of DNA instead of $52 \mathrm{pg}$, it is not that species. Again it might be an undescribed species.

5. And then there are tantalizing measurements such $45.3 \mathrm{pg}$ (instead of $56.7 \mathrm{pg}$ ) for a large "T. biebersteiniana" growing in cultivated fields west of Quba, northern Azerbaijan and $51.9 \mathrm{pg}$ (instead of $40.1 \mathrm{pg}$ ) for a " $T$. tetraphylla" from Toragyr Range, ZO Kazakhstan, that cannot be explained by ploidy.

Acknowledgments I would like to thank Mr. W. Lemmers and Mr. J. de Groot for their excellent advice, based on their hands-on experience. Mr. E. Boon of Iribov, Heerhugowaard, is thanked for keeping the flow cytometry apparatus going. J. F. Veldkamp is thanked for his expert advice on all nomenclatural matters and for the careful reading of the manuscript. The contribution of plant material by the donors listed above is gratefully acknowledged.

Open Access This article is distributed under the terms of the Creative Commons Attribution Noncommercial License which permits any noncommercial use, distribution, and reproduction in any medium, provided the original author(s) and source are credited. 


\section{Appendix: New species and combinations}

A detailed explanation of the infrageneric nomenclature of Tulipa as mentioned above can be found in Veldkamp and Zonneveld (submitted).

New species Tulipa lemmersii Zonn., A. Peterse, J. de Groot, sp. nov.

Planta erecta, parva. Bulbus 2-2.5 cm diametro; tunicae brunneae, tenerae, intus e basi ad apicem pilis inspersae.

Caulis glaber, uniflorus, parte supraterranea c. $7 \mathrm{~cm}$.

Folia 3, undulata, glabra, marginem non ciliatum, inferiore $15 \times 3 \mathrm{~cm}$, supra $15 \times 1.5 \mathrm{~cm}$.

Perianthi segmenta intus lutea, extus pallidiora coccinea suffusa, $3.5 \times 2 \mathrm{~cm}$, interiora obovata, exteriora ovata. Staminum filamenta lutea, glabra, $8 \times 1.5 \mathrm{~mm}$; antherae nigrae $6 \mathrm{~mm}$; pollen ferrugineum vel isabellinum. Ovarium $10 \mathrm{~mm}$ longum, viride, stigma sessile lutea, $3 \mathrm{~mm}$. T. lemmersii a T. iliensis, T. ferganica, T. anisophylla, T. tetraphylla caulis glaber et uniflorus differt. Ceterum speciorum sectio Kolpakowskianae floribus et rubris et luteis differt. Species diploidea, optime distinguitur a toto specie sectio Kolpakowskianae infimus DNA contento nucleorum $36 \mathrm{pg}$.

Distributia in Mashad Pass, Chimkent, Kazakhstan.

Type Herbarium Lugdunum Batavorum deposit.

Tulip erect, small. Bulb 2-2.5 cm in diam.; tunic thin, brown, at base and top lightly hairy.

Stem glabrous, single-flowered, aboveground part $7 \mathrm{~cm}$, excluding flower of $3.5 \mathrm{~cm}$. Leaves 3 , glabrous and without cilia, lower leaf $15 \times 3 \mathrm{~cm}$, upper leaf $15 \times 1.5 \mathrm{~cm}$.

Inner tepals $3.5 \times 2 \mathrm{~cm}$, obovate, outer tepals ovate, flower yellow with a red hue on the outside. Filaments yellow, glabrous, $8 \times 1.5 \mathrm{~mm}$; anthers $6 \mathrm{~mm}$, black with light brown pollen. Ovarium $1 \mathrm{~cm}$ high, green, with sitting yellow stigma.

T. lemmersii differs from $T$. iliensis, $T$. ferganica, T. anisophylla, and T. tetraphylla by its glabrous stem, thin tunic, and single flower. It can be distinguished from the other species of section Kolpakowskianae as the latter have mainly both red flowers and yellow flowers. It is a diploid species that has the lowest nuclear DNA content (36 pg) of all species of section Kolpakowskianae.

The tulip was collected by A. Peterse on top of the steep cliffs in the Mashad Pass. He accompanied a tulip expedition organized by $\mathrm{W}$. Lemmers, the eminent connoisseur of the wild middle Asiatic tulip flora, to whom this tulip is dedicated.

Tulipa orphanidea subsp. doerfleri (Gand.) Zonn., comb. et stat. nov. Basionym: Tulipa doerfleri Gand., Fl. Cretica 102. 1916.

Note: The Flora cretica is a lithographed manuscript and is a valid publication (Art. 30.2; the work is not listed in
Appendix V of the ICBN on the Opera utique oppressa for Gandoger).

Tulipa orphanidea Boiss. ex Heldr. subsp. whittalii (A.D. Hall) Zonn., comb. et stat. nov.

$\equiv$ Tulipa whittallii Elwes ex W.C.F. Newton. J Linn Soc Bot 47:341, 342. 1926, nom. inval., sine descr. $\equiv$ Tulipa whittallii A.D. Hall, Book Tulip.: 70. 1929; Gen. Tulipa, 64, t. 5. 1940; Bot Mag Lond 164:t. 9649. 1943. 三 Tulipa orphanidea Boiss. ex Heldr. var. whittallii (A.D. Hall) Boiss. ex Dykes, Not. Tulip Sp.: 34, t. 8.1930 (sine ref., see note) $\equiv$ Tulipa orphanidea (var.) whittallii A. Baker, J. Roy. Hort Soc Lond 56:242, 243. 1931 ("orphanidesii"). Type: Not indicated.

Dykes (1930) refers to a T. orphanidea " var. whittallii" and his plate is subtitled T. orphanidea var. whittallii Boiss. No. 1560. We suspect that "Boiss." is a printing error, for Boissier was the author of the species, but we have found no reference to any "whittallii" so named by him. Dykes gives no reference to previous uses of or descriptions for this variety, but the use of the epithet suggests that Art. 33.3 applies here and that Hall is the author in parentheses. We have only seen the relevant pages and surmise that 1560 may be the accession number.

Tulipa saxatilis subsp. bakeri (A.D. Hall) Zonn. comb. et stat. nov. Basionym: T. bakeri A.D. Hall J Bot 76: 316 (1938).

Tulipa korolkowii forma rosea (Vved.) Zonn. comb. et stat. nov.

Basionym T. rosea Vved. Opred Rast Sred Azii 2: 319 (1971).

Flowers are pink instead of red.

\section{References}

Baker JG (1883) The species of Tulipa. Gard Chron 19:691

Bennett MD (1972) Nuclear DNA content and minimum generation time in herbaceous plants. Proc R Soc Lond 181:109-135

Bennett MD, Leitch IJ (2004) Plant DNA $C$-values database (release 4.0, 2005). http://data.kew.org/cvalues/homepage.html

Bennetzen JL, Kellog EA (1997) Do plants have a one way ticket to genomic obesity? Plant Cell 9:1509-1514

Blakey D, Vosa CG (1962) Heterochromatin and chromosome variation in cultivated species of Tulipa subg. Leiostemones (Liliaceae). Plant Syst Evol 139:163-178

Boissier E (1882) Flora Orientalis 5:191-201. Geneva

Booy G, Van Raamsdonk LWD (1998) Variation in the enzyme esterase within and between Tulipa species; usefulness for the analysis of genetic relationships at different taxonomic levels. Bioch Syst Ecol 26:199-124

Botschantzeva ZP (1962) Tulips: taxonomy, morphology, cytology, phytogeography, and physiology. English translated edition by HQ Varekamp (1982). Balkema, Rotterdam

Britton NL, Brown A (1913) An illustrated flora of the northern United States and Canada, 2nd edn. Charles Scribner's Sons, New York 
Dasgupta S, Deb DB (1985) Taxonomic revision of the genus Tulipa L. in India and adjoining regions. Candollea 40:157-172

De Mol WE (1928) Zusammenfassung der zytologischen und genetischen Ergebnisse des Versuchs zur Duplizierung und Quadruplizierung von Sexualkernen bei Hyazinthen und Tulpen. Z Indukt Abstamm-Vererbungsl 48:145-148

Furse P (1965) Iran and Afghanistan 1964. J R Hort Soc 90:462-475, 506-509

Furse P (1966) Iran and Afghanistan 1964 (cont.). J R Hort Soc 91:18-26

Gabrielian E (1966) Generis Tulipa L. Species nova e Transcaucasia. Nov Syst Pl Vasc Ac Sci URSS 3:8-40

Govaerts R (2008) World checklist series, RBG Kew, UK: genus Tulipa.http://apps.kew.org/wcsp/qsearch.do

Greilhuber J (1979) Evolutionary changes of DNA and heterochromatin amounts in the Scilla bifolia Group (Liliaceae). Plant Syst Evol Suppl 2:263-280

Greilhuber J (1998) Intraspecific variation in genome size: a critical reassessment. Ann Bot 82:27-35

Greilhuber J (2005) Intraspecific variation in genome size in angiosperms: identifying its existence. Ann Bot 95:91-98

Grey-Wilson C, Mathews V (1980) Tulipa L. In: Tutin et al (eds) Flora Europaea, vol 5. Cambridge University Press, Cambridge, pp 28-31

Hall AD (1940) The genus Tulipa. The Royal Horticultural Society, London

Hewer TF (1974) Some observations on tulips in Iran and Afghanistan. Lilies and other Liliaceae 1974. Royal Horticultural Society, London, pp 70-74

Hitchcock AS, Green ML (1929) Standard species of Linnean genera of Phanerogamae. In: International Botanical Congress, Cambridge 1930. Nomenclature. Proposals by British Botanists. Wyman, London, pp 110-199

Hoog MH (1947) New bulbous and tuberous rooted plants. Van Tubergen, Haarlem

Hoog MH (1973) On the origin of Tulipa. Lilies and other Liliaceae. Royal Horticultural Society, London

Ivaschenko A (2005) Tulips of Kazakhstan Shell Kazakhstan development. BV Printing House: "Two Kapitals". ISBN: 9965-9593-3-1

Jarvis C, Barrie FR, Allan DM, Reveal JL (1992) Seventy-two proposals for the conservation of types of selected Linnaean generic names, the report of Subcommittee 3C on the lectotypification of Linnaean generic names. Taxon 41:552-583

Jarvis C, Barrie FR, Allan DM, Reveal JL (1993) A list of Linnean generic names and their types. Regnum vegetabile, vol 127. Koeltz, Koenigstein

Kroon GH, Jongerius MC (1986) Chromosome numbers of Tulipa species and the occurrence of hexaploidy. Euphytica 35:73-76

Kroon GH, Van Eijk JP (1977) Polyploidy in tulips (Tulipa L.). The occurrence of diploid gametes. Euphytica 26:33-66

Leitch I, Chase MW, Bennett MD (1998) Phylogenetic analysis of DNA $C$-values provides evidence for a small ancestral genome size in flowering plants. Ann Bot 82(suppl A):85-94

Levier E (1884) Les Tulipes de l'Europe. Bull Soc Sci Nat Neuchatel $14: 201-312$

Linnaeus C (1737) Hortus cliffortianus, 1st edn. Amsterdam, p 128

Linnaeus C (1753) Species plantarum. Salvius, Sockholm, p 306

Marais W (1984) Tulipa L. In: Davis PH (ed) Flora of Turkey and the East Aegean Islands, vol 8. University Press, Edinburgh, pp 302-311

Mathew B (2005) A review of Tulipa species. Daffodil Snowdrop Tulip Yearbook 2004-2005, pp 48-54

Mouterde P (1966) Nouvelle flore du Liban et de la Syrie. Dar El-Machreq, Beyrouth

Newton WCF (1927) Chromosome studies in Tulipa and some related genera. J Linn Soc London 47:339
Ohri D (1998) Genome size variation and plant systematics. Ann Bot 82(suppl A):750-812

Phillips R, Rix M (1981) The bulb book. Pan Books, London

Plavcova O (1969) Die Chromosomenzahlen bei Gartentulpen. Acta Pruhon 21:1-20

Pratov UP, Sharipov A, Ashurmetov OA, Tadjibaev KS (2006) Tulips of the western Tien Shan. Ac Sc Rep Uzbekistan, Tasskent

Rudall PJ, Stobart KL, Hong WP, Conran JG, Furness CA, Kite GC, Chase MW (2000) Consider the lilies: systematics of Liliales. In: Wilson KL, Morrison DA (eds) Monocots. Systematics and Evolution. CSIRO, Melbourne

Stork A (1984) Tulipes sauvages et cultivées. Série documentaire 13. Conservatoire et jardin botaniques, Geneva

Tiersch TR, Chandler RW, Wachtel SSM, Ellias S (1989) Reference standards for flow cytometry and application in comparative studies of nuclear DNA content. Cytometry 10:706-710

Van Eyk JP, Van Raamsdonk LWD, Eikelboom W, Bino RJ (1991) Interspecific crosses between Tulipa gesneriana cultivars and wild Tulipa species: a survey. Sex Pl Reprod 4:1-5

Van Raamsdonk LWD (1992) Biosystematic studies in Tulipa subgenus Eriostemones. Plant Syst Evol 179:27-41

Van Raamsdonk LWD, De Vries T (1995) Species relationships and taxonomy in Tulipa subgenus Tulipa (Liliaceae). Plant Syst Evol 195:13-44

Van Raamsdonk LWD, Van Eyk JP, Eikelboom W (1995) Crossability analysis in subgenus Tulipa of the genus Tulipa. Bot $\mathbf{J}$ Linn Soc 117-1:147-158

Van Raamsdonk LWD, Eikelboom W, De Vries T, Straathof PT (1997) The systematics of the genus Tulipa L. In: Lilien-Kipnis $\mathrm{H}$, Halevy AH, Borochov A (eds) Proceedings of the international symposium on flower bulbs. Acta Hort (ISHS) 430:821-828

Van Scheepen J (1996) Classified list and international register of tulip names. KAVB, Hillegom, The Netherlands

Vvedenskii AI (1935) Tulipa. In: Komarov VL (ed) Flora of the USSR, vol 4, English edn (1968). Izd An SSR, Leningrad, pp 246-280

Wilford R (2006) Tulips, species and hybrids for the gardener. Timber Press, Portland

Woods MW, Bamford R (1937) Chromosome morphology and number in Tulipa. Am J Bot 24:174-184

Zeiling AE, Schouten HP (1968) Polyploidy in garden tulips. I. A survey of Tulipa varieties for polyploids. Euphytica 17:252-264

Zonneveld BJM (2001) Nuclear DNA contents of all species of Helleborus discriminate between species and sectional divisions. Plant Syst Evol 229:125-130

Zonneveld BJM (2003) The systematic value of nuclear DNA content in Clivia. Herbertia 57:41-47

Zonneveld BJM (2008) The systematic value of nuclear DNA content for all species of Narcissus L. (Amaryllidaceae). Plant Syst Evol 275:109-132

Zonneveld BJM, Duncan GD (2003) Taxonomic implications of genome size and pollen color and vitality for species of Agapanthus L'Heritier (Agapanthaceae). Plant Syst Evol 241:115-123

Zonneveld BJM, Jaarsveld EJ (2005) Taxonomic implications of genome size for all species of the genus Gasteria Duval (Aloaceae). Plant Syst Evol 251:217-227

Zonneveld BJM, Duncan GD (2006) Genome size for species of Nerine Herb. (Amaryllidaceae) and its evident correlation with growth cycle, leaf width and other morphological characters. Plant Syst Evol 257:251-260

Zonneveld BJM, Van Iren F (2001) Genome size and pollen viability as taxonomic criteria: application to the genus Hosta. Plant Biol 3:176-185

Zonneveld BJM, Grimshaw JM, Davis AP (2003) The systematic value of nuclear DNA content in Galanthus. Plant Syst Evol 241:89-102 